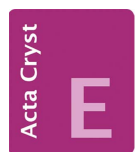

CRYSTALLOGRAPHIC COMMUNICATIONS

ISSN 2056-9890

Received 23 March 2017

Accepted 30 March 2017

Edited by P. C. Healy, Griffith University, Australia

Keywords: crystal structure; methacrylate; dimethoxybenzene; quinone; hydrogen bonds; $\mathrm{C}-\mathrm{H} \cdots \pi$ contacts.

CCDC references: 1541065; 1541064

Supporting information: this article has supporting information at journals.iucr.org/e

\section{Crystal structures of the polymer precursors 3-(2,5-dimethoxy-3,4,6-trimethylphenyl)propyl methacrylate and 3-(2,4,5-trimethyl-3,6-dioxo- cyclohexa-1,4-dienyl)propyl methacrylate}

Shailesh K. Goswami, Lyall R. Hanton, C. John McAdam, Stephen C. Moratti and Jim Simpson*

Department of Chemistry, University of Otago, PO Box 56, Dunedin, New Zealand. *Correspondence e-mail: jsimpson@alkali.otago.ac.nz

The closely related title compounds, 3-(2,5-dimethoxy-3,4,6-trimethylphenyl)propyl methacrylate, $\mathrm{C}_{18} \mathrm{H}_{26} \mathrm{O}_{4}(\mathrm{I})$, and 3-(2,4,5-trimethyl-3,6-dioxocyclohexa1,4-dienyl)propyl methacrylate, $\mathrm{C}_{16} \mathrm{H}_{20} \mathrm{O}_{4}$ (II), are monomers suitable for the preparation of redox polymers. They consist of a propylmethacrylate group and three methyl substituents on dimethoxybenzene and quinone cores, respectively. Both crystal structures feature weak $\mathrm{C}-\mathrm{H} \cdots \mathrm{O}$ hydrogen bonds and $\mathrm{C}-$ $\mathrm{H} \cdot \cdots \pi$ (ring) contacts between methyl groups and the six-membered rings.

\section{Chemical context}

The title compounds, (I) and (II), were synthesised as part of our continuing interest in redox polymers and electrochemical actuators (Dana et al., 2007; McAdam et al., 2008; Goswami et al., 2013, 2015). Redox-active polymers containing 2,2,6,6tetramethylpiperidin-1-oxyl-4-yl (TEMPO) and ferrocene as pendant groups are well documented (Gracia \& Mecerreyes, 2013; Tamura et al., 2008; Schattling et al., 2014). In contrast, polymers with pendant quinone units are less well explored (Hodge \& Gautrot, 2009; Häupler et al., 2014). Reasons for this include their free-radical-scavenging properties in free radical polymerization (FRP), and the incompatibility of the quinone carbonyl groups in typical living polymerization such as anionic or cationic polymerization. In previous work (Goswami et al., 2013) we successfully demonstrated that steric hindrance by alkyl groups around a quinone unit prevents radical addition to the ring or the carbonyl oxygen atom, thus enabling FRP synthesis of homo- and co-polymers of quinone-appended methacrylate monomers.

\section{Structural commentary}

Compound (I), a tetra-alkylated p-dimethoxybenzene is shown in Fig. 1. The methoxy substituents are in the typical trans conformation (Wickramasinhage et al., 2016; Wiedenfeld et al., 2003; Wieczorek et al., 1975) with a C111-O1...O4C41 torsion angle of approximately $179.24^{\circ}$. Three methyl groups and a propyl methacrylate occupy the other four sites on the benzene ring. Compound (II), shown in Fig. 2, is the quinone analogue of (I). As expected, the oxidation destroys the aromaticity of the six-carbon ring, reflected in a shortening 
of $\mathrm{C} 2-\mathrm{C} 3$ and $\mathrm{C} 5-\mathrm{C} 6$ and a lengthening of the other ring C-C bonds (Allen et al., 1987).

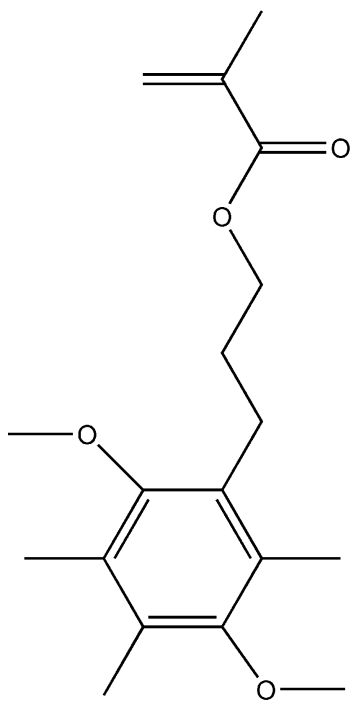

(I)

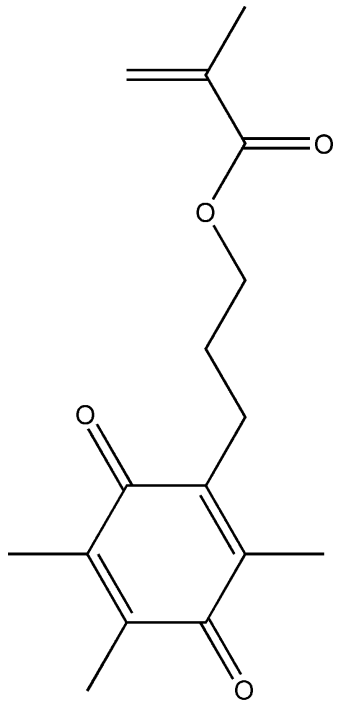

(II)
The spatial arrangement of the ring substituents and the propyl methacrylate moiety is remarkably similar to that observed for (I). In particular, the torsional geometry of the vinyl and carbonyl components of the methacrylate groups of both (I) and (II) display the typical $s$-trans preference

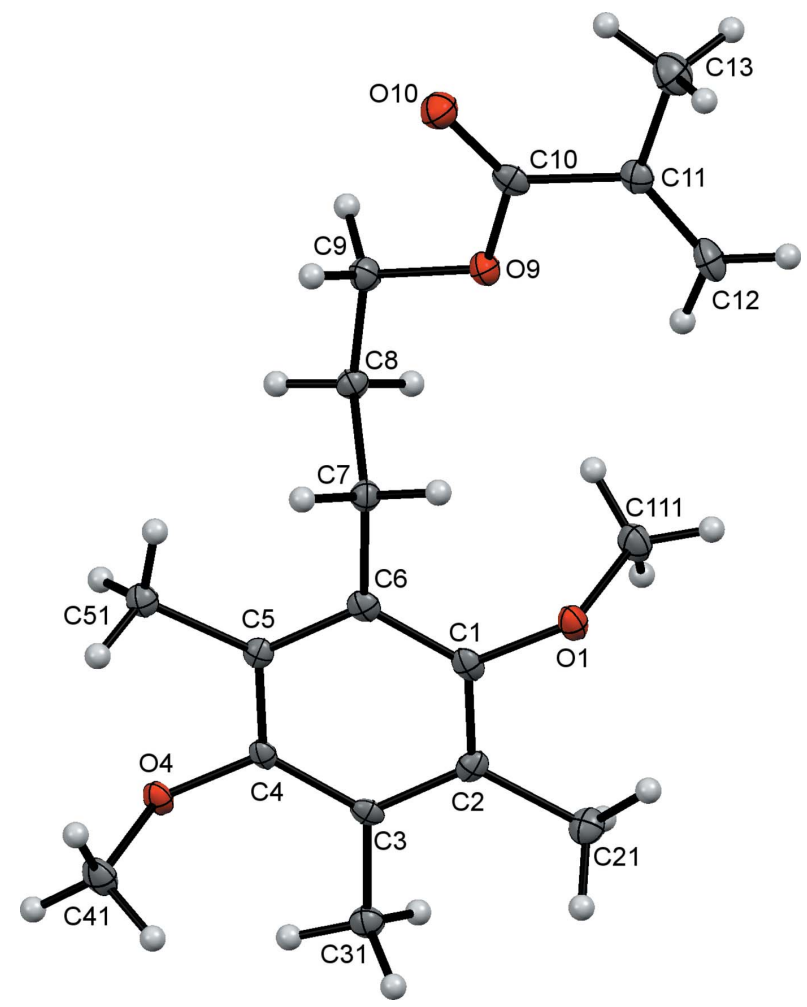

Figure 1

The molecular structure of compound (I), with displacement ellipsoids drawn at the $50 \%$ probability level.
Table 1

Hydrogen-bond geometry $\left(\AA,^{\circ}\right)$ for (I).

$\mathrm{Cg}$ is the centroid of the $\mathrm{C} 1-\mathrm{C} 6$ benzene ring

\begin{tabular}{lllll}
\hline$D-\mathrm{H} \cdots A$ & $D-\mathrm{H}$ & $\mathrm{H} \cdots A$ & $D \cdots A$ & $D-\mathrm{H} \cdots A$ \\
\hline $\mathrm{C} 8-\mathrm{H} 8 B \cdots \mathrm{O}^{\mathrm{i}}$ & 0.99 & 2.58 & $3.456(4)$ & 147 \\
$\mathrm{C} 12-\mathrm{H} 12 B \cdots \mathrm{O}^{\mathrm{ii}}$ & 0.95 & 2.50 & $3.388(4)$ & 157 \\
$\mathrm{C} 21-\mathrm{H} 21 A \cdots 1^{\mathrm{iii}}$ & 0.98 & 2.67 & $3.614(5)$ & 161 \\
$\mathrm{C} 41-\mathrm{H} 41 A \cdots \mathrm{O}^{\mathrm{iv}}$ & 0.98 & 2.66 & $3.590(5)$ & 159 \\
$\mathrm{C} 51-\mathrm{H} 51 E \cdots 4^{\mathrm{v}}$ & 0.98 & 2.65 & $3.541(4)$ & 151 \\
$\mathrm{C} 7-\mathrm{H} 7 B \cdots C g^{\mathrm{v}}$ & 0.99 & 2.97 & $3.709(4)$ & 134 \\
$\mathrm{C} 31-\mathrm{H} 31 C \cdots C g^{\mathrm{iii}}$ & 0.98 & 2.85 & $3.693(4)$ & 148 \\
\hline
\end{tabular}

Symmetry codes: (i) $-x+1,-y,-z+2$; (ii) $x+\frac{1}{2},-y+\frac{1}{2}, z+\frac{1}{2}$; (iii) $x-1, y, z$; (iv) $x-1, y, z-1 ;(\mathrm{v}) x+1, y, z$.

(McAdam et al., 2015). Predictably, both the benzene and quinone ring systems (C1-C6) and the attached atoms (O1, C21, C31, O4, C51 and C7) are nearly planar, with r.m.s. deviations from the mean planes of 0.0377 and $0.0158 \AA$, respectively.

\section{Supramolecular features}

\subsection{Crystal packing for (I)}

In the crystal structure of (I), $\mathrm{C} 21-\mathrm{H} 21 A \cdots \mathrm{O} 1$ and $\mathrm{C} 51-$ $\mathrm{H} 51 E \cdots \mathrm{O} 4$ hydrogen bonds form chains of molecules along

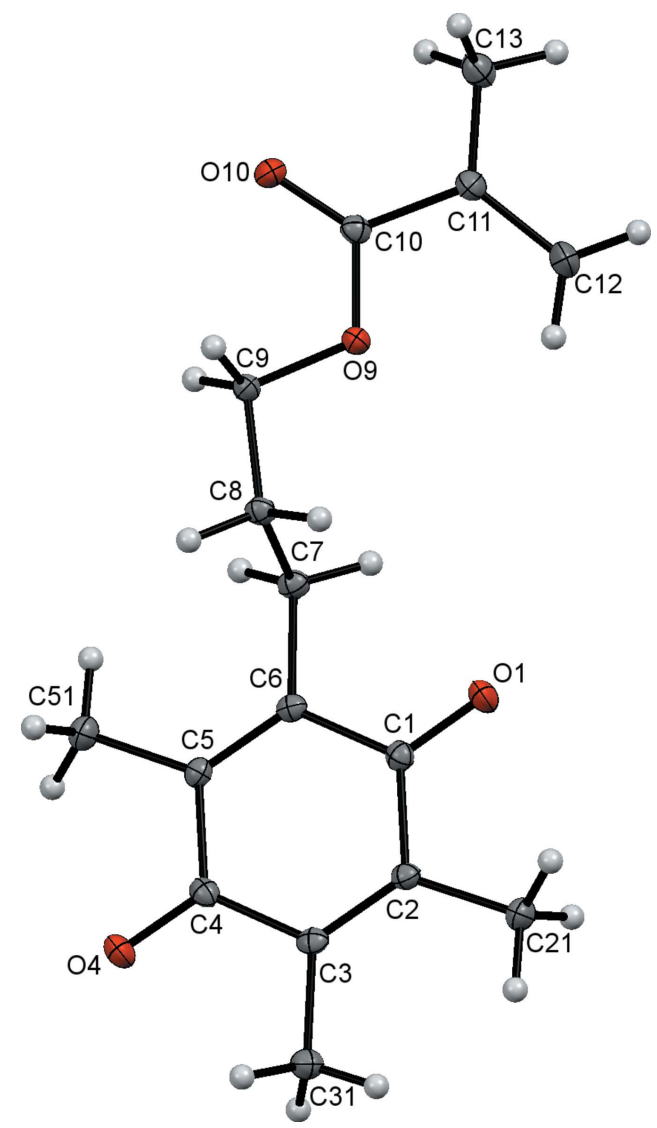

Figure 2

The molecular structure of compound (II), with displacement ellipsoids drawn at the $50 \%$ probability level. 


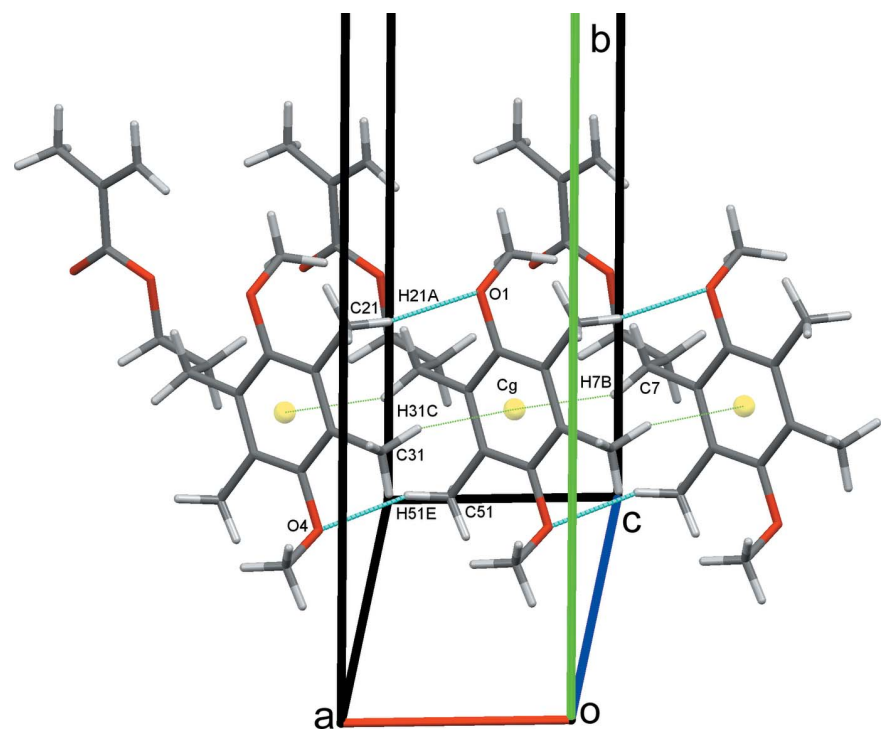

Figure 3

Chains of molecules of (I) along the $a$-axis direction.

the $a$-axis direction. The chains are reinforced by $\mathrm{C} 7-$ $\mathrm{H} 7 \mathrm{~B} \cdots C \mathrm{Cg}$ and $\mathrm{C} 31-\mathrm{H} 31 \mathrm{C} \cdots C g$ contacts $(C g$ is the centroid of the $\mathrm{C} 1-\mathrm{C} 6$ ring) between methyl and methylene group hydrogen atoms and the aromatic ring, Table 1 and Fig. 3. $\mathrm{C} 12-\mathrm{H} 12 B \cdots \mathrm{O} 1$ and $\mathrm{C} 41-\mathrm{H} 41 A \cdots \mathrm{O} 10$, hydrogen bonds link these chains into a sheet, two-molecules thick, that lies parallel to the $a c$ plane (010), Fig. 4. Extension to a threedimensional structure is completed by $\mathrm{C} 8-\mathrm{H} 8 B \cdots \mathrm{O} 4$ inversion dimers. These form $R_{2}^{2}(14)$ rings and link pairs of doublelayer sheets, stacking molecules along the $a$-axis direction, Fig. 5.

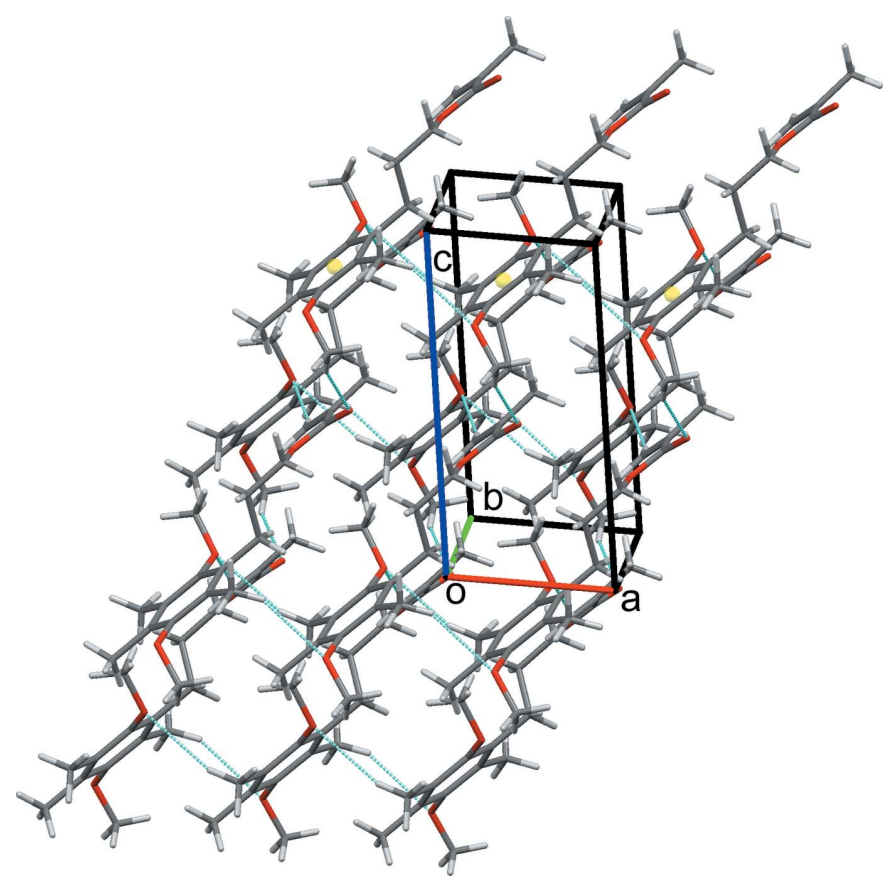

Figure 4

A double sheet of molecules of (I) in the ac plane.

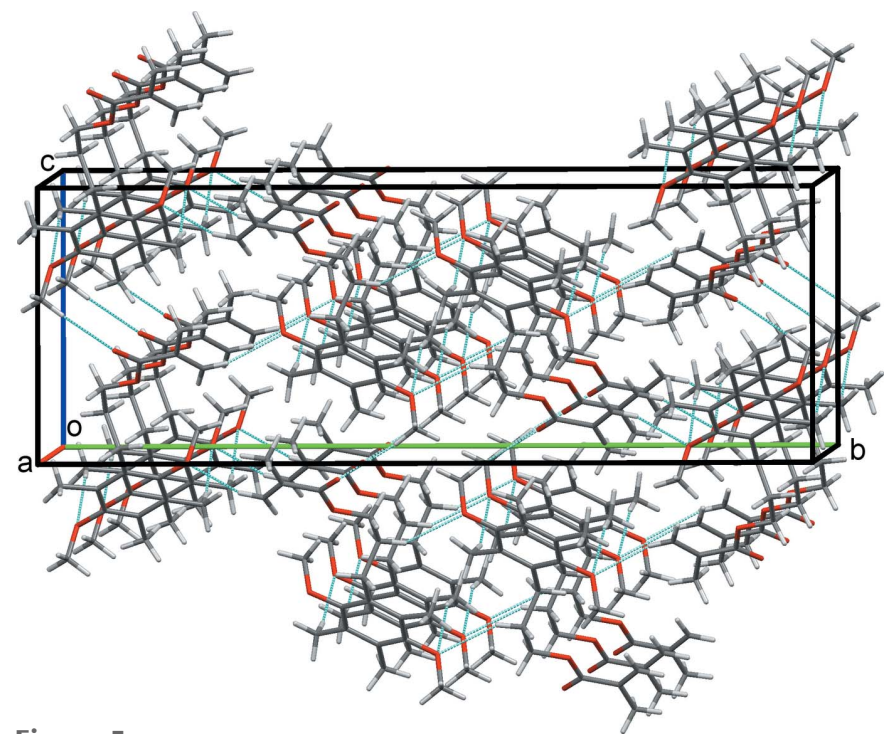

Figure 5

Overall packing for (I) viewed along the $a$-axis direction.

\subsection{Crystal packing for (II)}

For (II), an extensive series of $\mathrm{C}-\mathrm{H}$. . O hydrogen bonds and a $\mathrm{C}-\mathrm{H} \cdots \pi$ (ring) contact generate the three-dimensional structure. These contacts include O10 acting as a trifurcated acceptor; C9-H9B ..OO10 hydrogen bonds, supported by $\mathrm{C} 31-\mathrm{H} 31 \mathrm{~B} \cdots \mathrm{Cg}$ contacts $(\mathrm{Cg}$ is the centroid of the $\mathrm{C} 1-\mathrm{C} 6$ ring), Table 2, form chains along the $a$-axis direction, Fig. 6 . The other two components of the trifurcate, the inversionrelated $\mathrm{C} 9-\mathrm{H} 9 A \cdots \mathrm{O} 10$ and $\mathrm{C} 51-\mathrm{H} 51 B \cdots \mathrm{O} 10$ hydrogen bonds form $R_{2}^{2}(10)$ and $R_{2}^{2}(20)$ rings, respectively. A third inversion dimer results from $\mathrm{C} 12-\mathrm{H} 12 A \cdots \mathrm{O} 1$ contacts and forms $R_{2}^{2}(22)$ rings. $\mathrm{O} 4$ acts as a bifurcated acceptor, forming $\mathrm{C} 21-\mathrm{H} 21 A \cdots \mathrm{O} 4$ and $\mathrm{C} 31-\mathrm{H} 31 C \cdots \mathrm{O} 4$ hydrogen bonds that enclose $R_{2}^{1}(7)$ rings, completing an extensive sheet of molecules parallel to (105), Fig. 7. This eclectic array of contacts combine to produce a three-dimensional network with molecules stacked along the $a$ axis, Fig. 8 .

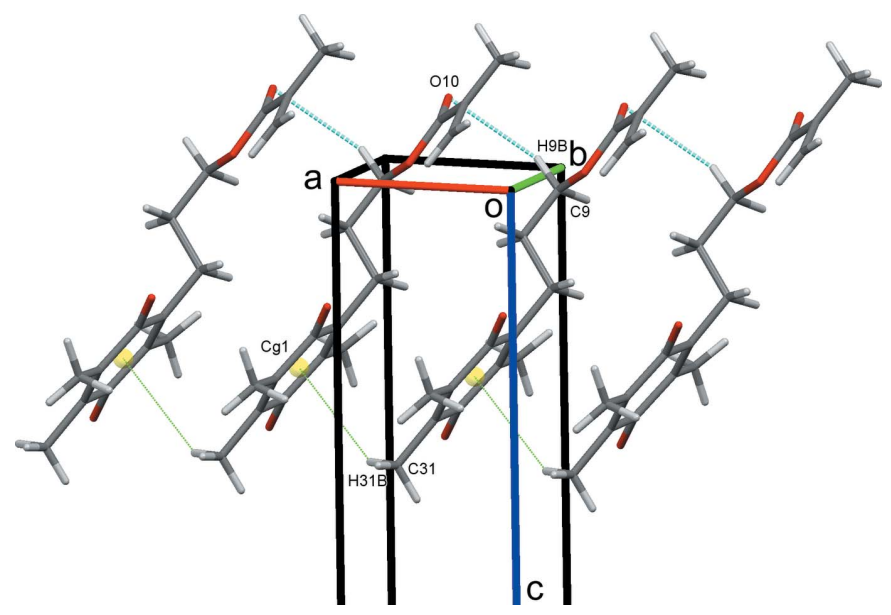

Figure 6

Chains of molecules of (II) formed along $a$. 
Table 2

Hydrogen-bond geometry $\left(\AA,^{\circ}\right)$ for (II).

$\mathrm{Cg}$ is the centroid of the $\mathrm{C} 1-\mathrm{C} 6$ ring.

\begin{tabular}{lllll}
\hline$D-\mathrm{H} \cdots A$ & $D-\mathrm{H}$ & $\mathrm{H} \cdots A$ & $D \cdots A$ & $D-\mathrm{H} \cdots A$ \\
\hline $\mathrm{C} 9-\mathrm{H} 9 A \cdots \mathrm{O} 10^{\mathrm{i}}$ & 0.99 & 2.72 & $3.624(3)$ & 153 \\
$\mathrm{C} 9-\mathrm{H} 9 B \cdots \mathrm{O} 10^{\text {ii }}$ & 0.99 & 2.70 & $3.595(3)$ & 150 \\
$\mathrm{C}^{\mathrm{iii}}-\mathrm{H} 12 A \cdots \mathrm{O} 1^{\text {ii }}$ & 0.95 & 2.53 & $3.422(3)$ & 156 \\
$\mathrm{C} 21-\mathrm{H} 21 A \cdots \mathrm{O} 4^{\text {iv }}$ & 0.98 & 2.52 & $3.455(3)$ & 161 \\
$\mathrm{C} 31-\mathrm{H} 31 C \cdots \mathrm{O} 4^{\text {iv }}$ & 0.98 & 2.68 & $3.638(3)$ & 166 \\
$\mathrm{C} 51-\mathrm{H} 51 B \cdots \mathrm{O} 10^{\mathrm{v}}$ & 0.98 & 2.67 & $3.510(3)$ & 144 \\
$\mathrm{C} 31-\mathrm{H} 31 B \cdots C g^{\text {ii }}$ & 0.98 & 2.95 & $3.534(3)$ & 119 \\
\hline
\end{tabular}

Symmetry codes: (i) $-x-1,-y+1,-z$; (ii) $x+1, y, z$; (iii) $-x-1,-y,-z$; (iv) $-x+\frac{3}{2}, y-\frac{1}{2},-z+\frac{1}{2} ;(\mathrm{v})-x,-y+1,-z$.

\section{Database survey}

A search of the CSD (Version 5.37 November 2015 with three updates; Groom et al., 2016) revealed a surprising degree of exclusivity for both of the title compounds. A search for the 2,5-dimethoxy-3,4,6-trimethylphenyl segment of (I) produced only two hits, our earlier report of the precursor 2,5-dimethoxy-3,4,6-trimethylbenzaldehyde (Wickramasinhage et al., 2016) and the dimer bis(2,5-dimethoxy-3,4,6-trimethylphenyl)methane (Wiedenfeld et al., 2003). A search for the corresponding quinone ring system was even less productive, with octamethyl-1,4-cyclohexanedione the only related structure (Hoffmann \& Hursthouse, 1976). Structures containing the propyl methacrylate moiety were similarly scarce, with the fullerene derivative 4-(6,9,12,15,18-pentamethyl- $\mathrm{C}_{60}$ fulleren1-yl)butyl methacrylate dichloromethane solvate (Matsuo et al., 2009) and a tungsten polyphosphate derivative (Hasegawa et al., 2007) the only hits.

\section{Synthesis and crystallization}

The synthesis of (I) was accomplished in three steps (Fig. 9) from 6-hydroxy-5,7,8-trimethylchroman-2-one (III) (Goswami et al., 2011) as described below.

Methylation of 6-hydroxy-5,7,8-trimethylchroman-2-one (III): To a solution of (III) $(5 \mathrm{~g}, 24 \mathrm{mmol})$ and dry $\mathrm{K}_{2} \mathrm{CO}_{3}$ $(13.4 \mathrm{~g}, 97 \mathrm{mmol})$ in $\mathrm{MeOH}(50 \mathrm{~mL})$ was added MeI $(13.8 \mathrm{~mL}$, $97 \mathrm{mmol})$. The mixture was refluxed for $4 \mathrm{~h}$, filtered through celite, and solvent removed in vacuo to afford methyl 3-(2,5dimethoxy-3,4,6-trimethylphenyl)propanoate (IV) (5.5 g,

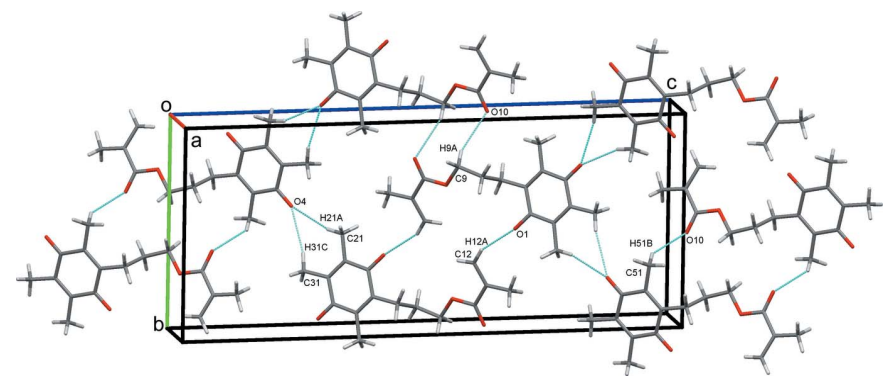

Figure 7

Sheets of molecules of (II) viewed along $a$.

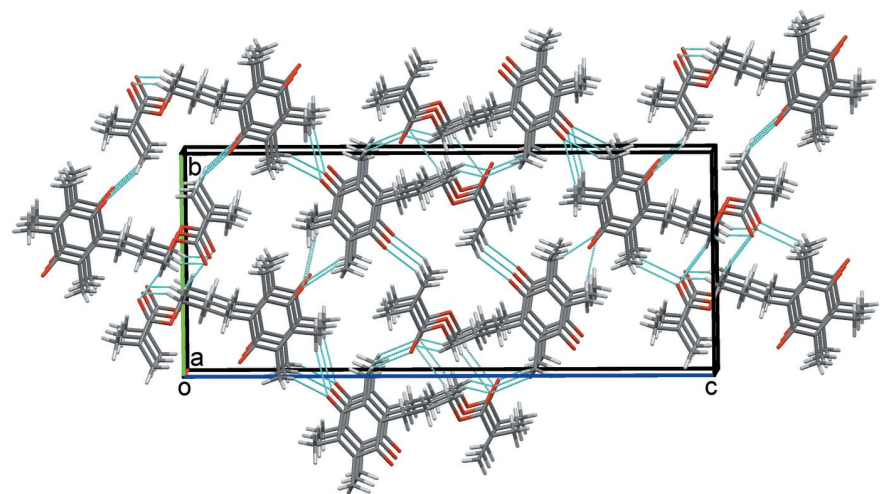

Figure 8

Overall packing for (II) viewed along the $a$-axis direction.

$85 \%)$ as a yellow liquid. MS calculated for $\left[\mathrm{C}_{15} \mathrm{H}_{22} \mathrm{NaO}_{4}\right]^{+}$: 289.1410. Found: 289.1391 (6.72 ppm). IR (KBr) $v_{\mathrm{C}=\mathrm{O}}$ : $1750 \mathrm{~cm}^{-1}$ (methyl ester). ${ }^{1} \mathrm{H}$ NMR $\left(\mathrm{CDCl}_{3}, \delta \mathrm{ppm}\right): 2.18(s$, $\left.6 \mathrm{H}, 2 \times \mathrm{Ar}-\mathrm{CH}_{3}\right), 2.24\left(s, 3 \mathrm{H}, \mathrm{Ar}-\mathrm{CH}_{3}\right), 2.49 \& 2.95[2 \times(t, J=$ $\left.\left.7.8 \mathrm{~Hz}, 2 \mathrm{H}, \mathrm{CH}_{2}\right)\right], 3.65\left(s, 3 \mathrm{H}\right.$, ester $\left.\mathrm{OCH}_{3}\right), 3.68 \& 3.71[2 \times$ $\left.\left(s, 3 \mathrm{H}, \mathrm{Ar}-\mathrm{OCH}_{3}\right)\right] .{ }^{13} \mathrm{C} \mathrm{NMR}\left(\mathrm{CDCl}_{3}, \delta \mathrm{ppm}\right): 12.3,12.8,13.0$, 23.0, 34.5, 51.5, 60.6, 61.1, 127.5, 128.4, 129.2, 130.4, 153.3, 174.0.

Reduction of methyl 3-(2,5-dimethoxy-3,4,6-trimethylphenyl)propanoate (IV): To a stirred suspension of $0.85 \mathrm{~g}$ ( $22 \mathrm{mmol}$ ) of $\mathrm{LiAlH}_{4}$ in $100 \mathrm{~mL}$ dry THF cooled to $273 \mathrm{~K}$ in ice a solution of $5.0 \mathrm{~g}(18.7 \mathrm{mmol})$ of (IV) in $100 \mathrm{~mL}$ THF was added dropwise. After the vigorous reaction subsided, the mixture was heated to reflux for $2 \mathrm{~h}$. Excess of the hydride was decomposed by careful addition of water, and the mixture was neutralized with acetic acid. To this was added $650 \mathrm{~mL}$ of saturated aq. $\mathrm{NH}_{4} \mathrm{Cl}$ solution. The organic layer was separated and the aqueous layer further extracted with $4 \times 150 \mathrm{~mL}$ portions of THF. The combined THF layers were dried over $\mathrm{MgSO}_{4}$ and solvent removed in vacuo. Recrystallization from $\mathrm{Et}_{2} \mathrm{O}$ gave $4.1 \mathrm{mg}$ (91\%) of 3-(2,5-dimethoxy-3,4,6-trimethylphenyl)propan-1-ol (V) as a white solid, m.p. 461-463 K please check. MS calculated for $\left.\mathrm{C}_{14} \mathrm{H}_{22} \mathrm{NaO}_{3}\right]^{+}: 261.1461$. Found: 246.1461 (0 ppm). IR (KBr) $v_{\mathrm{OH}}: 3425,3150 \mathrm{~cm}^{-1} \cdot{ }^{1} \mathrm{H}$ NMR $\left(\mathrm{CDCl}_{3}, \delta \mathrm{ppm}\right): 1.75\left(\mathrm{~m}, 2 \mathrm{H}, \mathrm{CH}_{2}\right), 2.09(\mathrm{~s}, 1 \mathrm{H} \mathrm{OH})$, $2.18\left(\mathrm{~s}, 6 \mathrm{H}, 2 \mathrm{Ar}-\mathrm{CH}_{3}\right), 2.23\left(\mathrm{~s}, 3 \mathrm{H}, \mathrm{Ar}-\mathrm{CH}_{3}\right), 2.75(\mathrm{t}, \mathrm{J}=$ $\left.7.3 \mathrm{~Hz}, 2 \mathrm{H}, \mathrm{Ar}-\mathrm{CH}_{2}\right), 3.52\left(\mathrm{t}, \mathrm{J}=6.6 \mathrm{~Hz}, 2 \mathrm{H}, \mathrm{CH}_{2}-\mathrm{OH}\right), 3.65$ \& $3.69\left[2\left(\mathrm{~s}, 3 \mathrm{H}, \mathrm{Ar}-\mathrm{OCH}_{3}\right)\right] \cdot{ }^{13} \mathrm{C} \mathrm{NMR}\left(\mathrm{CDCl}_{3}, \delta \mathrm{ppm}\right): 11.7$, 12.6, 12.8, 22.6, 32.0, 60.0, 61.0, 61.2, 127.4, 127.6, 128.6, 129.2, $130.4,153.0,153.5$.

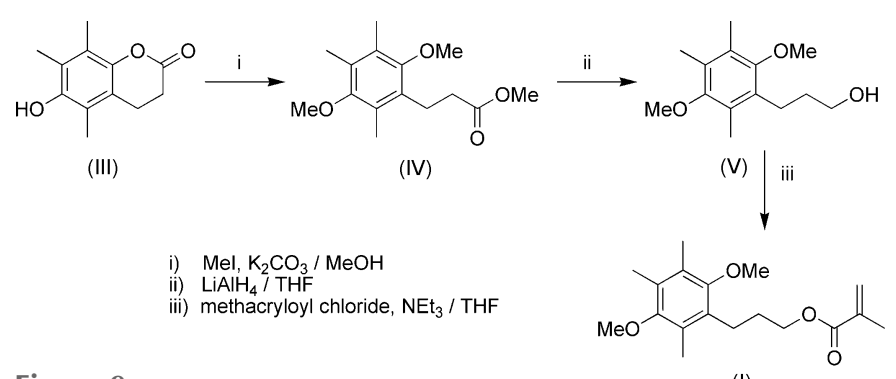

Figure 9

(I)

Steps involved in the synthesis of compound (I). 
Table 3

Experimental details.

(I)

Crystal data

Chemical formula

$M_{\mathrm{r}}$

Crystal system, space group

Temperature (K)

$a, b, c(\AA)$

$\beta\left({ }^{\circ}\right)$

$V\left(\AA^{3}\right)$

Z

Radiation type

$\mu\left(\mathrm{mm}^{-1}\right)$

Crystal size (mm)

Data collection

Diffractometer

Absorption correction

$T_{\min }, T_{\max }$

No. of measured, independent and

observed $[I>2 \sigma(I)]$ reflections

$R_{\text {int }}$

$\theta_{\max }\left({ }^{\circ}\right)$

$(\sin \theta / \lambda)_{\max }\left(\AA^{-1}\right)$

Refinement

$R\left[F^{2}>2 \sigma\left(F^{2}\right)\right], w R\left(F^{2}\right), S$

No. of reflections

No. of parameters

$\mathrm{H}$-atom treatment

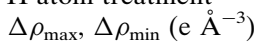

\author{
$\mathrm{C}_{18} \mathrm{H}_{26} \mathrm{O}_{4}$ \\ 306.39 \\ Monoclinic, $P 2_{1} / n$ \\ 91 \\ 5.1833 (7), 30.341 (4), 10.6339 (15) \\ $97.910(9)$ \\ $1656.4(4)$ \\ 4 \\ Mo $K \alpha$ \\ 0.09 \\ $0.65 \times 0.04 \times 0.04$
}

(II)

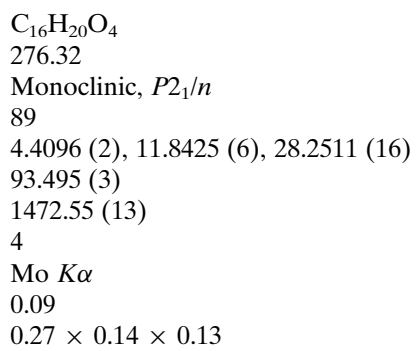

Bruker APEXII CCD area detector Multi-scan (SADABS; Bruker, 2013) $0.775,1.00$

$11585,1681,1254$

0.081

20.7

0.497

Bruker APEXII CCD area detector
Multi-scan $(S A D A B S$; Bruker, 2013)
$0.785,1.000$
$16398,2509,1774$

0.071
24.8
0.591

$0.049,0.138,1.04$
2509
185
H-atom parameters constrained
$0.34,-0.32$

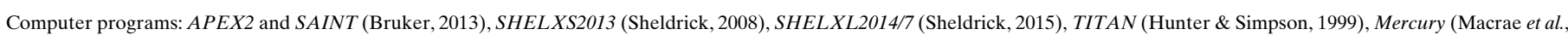
2008), enCIFer (Allen et al., 2004), PLATON (Spek, 2009) and publCIF (Westrip 2010).

Acylation of 3-(2,5-dimethoxy-3,4,6-trimethylphenyl)propan-1-ol (V): The alcohol (V) $(5.0 \mathrm{~g}, 21 \mathrm{mmol})$ was dissolved in $\mathrm{CH}_{2} \mathrm{Cl}_{2}(100 \mathrm{ml})$. $\mathrm{NEt}_{3}(2.2 \mathrm{~mL})$ was added and the solution stirred $30 \mathrm{~min}$ at $273 \mathrm{~K}$. Methacryloyl chloride $(2.4 \mathrm{~g}, 23 \mathrm{mmol})$ was added dropwise, stirred for $2 \mathrm{~h}$ under nitrogen at $273 \mathrm{~K}$ and then at room temperature for $4 \mathrm{~h}$. After extraction from $\mathrm{CH}_{2} \mathrm{Cl}_{2} / \mathrm{H}_{2} \mathrm{O}$ the organic layer was dried $\left(\mathrm{MgSO}_{4}\right)$ and solvent removed in vacuo. Purification using chromatography on $\mathrm{SiO}_{2}$ using petroleum ether/EtOAc (9:1) gave the colourless solid product (I), m.p. 395-397 K. MS calculated for $\left[\mathrm{C}_{18} \mathrm{H}_{26} \mathrm{NaO}_{4}\right]^{+}$: 329.1723. Found: 329.1709 (4.41 ppm). IR ( $\mathrm{KBr}) v_{\mathrm{C}=\mathrm{O}}: 1731 \mathrm{~cm}^{-1}$ (ester). ${ }^{1} \mathrm{H}$ NMR $\left(\mathrm{CDCl}_{3}, \delta\right.$ ppm): $1.89\left(m, 2 \mathrm{H}, \mathrm{CH}_{2}\right), 1.98\left(m, 3 \mathrm{H}, \mathrm{CH}_{3}\right), 2.19(s, 6 \mathrm{H}, 2 \times$ $\left.\mathrm{Ar}-\mathrm{CH}_{3}\right), 2.23\left(s, 3 \mathrm{H}, \mathrm{Ar}-\mathrm{CH}_{3}\right), 2.73(t, J=7.6 \mathrm{~Hz}, 2 \mathrm{H}, \mathrm{Ar}-$ $\left.\mathrm{CH}_{2}\right), 3.65 \& 3.68\left[2 \times\left(s, 3 \mathrm{H}, \mathrm{Ar}-\mathrm{OCH}_{3}\right)\right], 4.23(t, J=6.1 \mathrm{~Hz}$, $\left.2 \mathrm{H}, \mathrm{CH}_{2}\right), 5.57(m, 1 \mathrm{H},=\mathrm{CH}), 6.14(\mathrm{~m}, 1 \mathrm{H},=\mathrm{CH}) .{ }^{13} \mathrm{C} \mathrm{NMR}$ $\left(\mathrm{CDCl}_{3}, \delta \mathrm{ppm}\right): 12.2,12.9,13.1,18.6,24.1,29.5,60.3,61.1,65.0$, 125.4, 127.4, 128.2, 128.8, 131.4, 136.8, 153.20, 153.4, 167.8 . Crystals of (I) were obtained from a mixed $\mathrm{CH}_{2} \mathrm{Cl}_{2} /$ hexane solution $1 / 1 \mathrm{v} / \mathrm{v}$.

The synthesis of (II) has been reported previously (Goswami et al., 2013). Crystals were obtained from the slow evaporation of an $\mathrm{Et}_{2} \mathrm{O}$ solution.

\section{Refinement}

Crystal data, data collection and structure refinement details are summarized in Table 3. All $\mathrm{H}$ atoms were refined using a riding model with $d(\mathrm{C}-\mathrm{H})=0.95 \AA, U_{\text {iso }}=1.2 U_{\text {eq }}(\mathrm{C})$ for vinyl, $0.99 \AA, U_{\text {iso }}=1.2 U_{\text {eq }}(\mathrm{C})$ for $\mathrm{CH}_{2} \mathrm{H}$ atoms and $0.98 \AA$, $U_{\text {iso }}=1.5 U_{\text {eq }}(\mathrm{C})$ for $\mathrm{CH}_{3} \mathrm{H}$ atoms. The hydrogen atoms of the C13 and C51 methyl groups of (I) were equally disordered over two sites. Idealized disorder models were applied using AFIX123 in SHELXL2014/7. For (I), a low-angle reflection with $F_{\mathrm{o}}<<F_{\mathrm{c}}$, that may have been affected by the beam-stop, was omitted from the final refinement cycles.

\section{Acknowledgements}

We thank the NZ Ministry of Business, Innovation and Employment Science Investment Fund (grant No. UOOX1206) for support of this work and the University of Otago for the purchase of the diffractometer. JS thanks the Chemistry Department, University of Otago, for the support of his work.

\section{Funding information}

Funding for this research was provided by: NZ Ministry of Business, Innovation and Employment Science Investment Fund (award No. UOOX1206).

\section{References}

Allen, F. H., Johnson, O., Shields, G. P., Smith, B. R. \& Towler, M. (2004). J. Appl. Cryst. 37, 335-338.

Allen, F. H., Kennard, O., Watson, D. G., Brammer, L., Orpen, A. G. \& Taylor, R. (1987). J. Chem. Soc. Perkin Trans. 2, pp. S1-S19. 
Bruker (2013). APEX2, SAINT and SADABS. Bruker AXS Inc., Madison, Wisconsin, USA.

Dana, B. H., McAdam, C. J., Robinson, B. H., Simpson, J. \& Wang, H. (2007). J. Inorg. Organomet. Polym. Mater. 17, 547-559.

Goswami, S. K., Hanton, L. R., McAdam, C. J., Moratti, S. C. \& Simpson, J. (2011). Acta Cryst. E67, o1566-o1567.

Goswami, S. K., Hanton, L. R., McAdam, C. J., Moratti, S. C. \& Simpson, J. (2015). Acta Cryst. C71, 860-866.

Goswami, S. K., McAdam, C. J., Lee, A. M. M., Hanton, L. R. \& Moratti, S. C. (2013). J. Mater. Chem. A, 1, 3415-3420.

Gracia, R. \& Mecerreyes, D. (2013). Polym. Chem. 4, 2206-2214.

Groom, C. R., Bruno, I. J., Lightfoot, M. P. \& Ward, S. C. (2016). Acta Cryst. B72, 171-179.

Hasegawa, T., Shimizu, K., Seki, H., Murakami, H., Yoshida, S., Yoza, K. \& Nomiya, K. (2007). Inorg. Chem. Commun. 10, 11401144.

Häupler, B., Ignaszak, A., Janoschka, T., Jähnert, T., Hager, M. D. \& Schubert, U. S. (2014). Macromol. Chem. Phys. 215, 1250-1256.

Hodge, P. \& Gautrot, J. E. (2009). Polym. Int. 58, 261-266.

Hoffmann, H. M. R. \& Hursthouse, M. B. (1976). J. Am. Chem. Soc. 98, 7449-7450.

Hunter, K. A. \& Simpson, J. (1999). TITAN2000. University of Otago, New Zealand.
Macrae, C. F., Bruno, I. J., Chisholm, J. A., Edgington, P. R., McCabe, P., Pidcock, E., Rodriguez-Monge, L., Taylor, R., van de Streek, J. \& Wood, P. A. (2008). J. Appl. Cryst. 41, 466-470.

Matsuo, Y., Iwashita, A., Oyama, H. \& Nakamura, E. (2009). Tetrahedron Lett. 50, 3411-3413.

McAdam, C. J., Moratti, S. C. \& Simpson, J. (2015). Acta Cryst. C71, $1100-1105$.

McAdam, C. J., Nafady, A., Bond, A. M., Moratti, S. C. \& Simpson, J. (2008). J. Inorg. Organomet. Polym. Mater. 18, 485-490.

Schattling, P., Jochum, F. D. \& Theato, P. (2014). Polym. Chem. 5, 2536.

Sheldrick, G. M. (2008). Acta Cryst. A64, 112-122.

Sheldrick, G. M. (2015). Acta Cryst. C71, 3-8.

Spek, A. L. (2009). Acta Cryst. D65, 148-155.

Tamura, K., Akutagawa, N., Satoh, M., Wada, J. \& Masuda, T. (2008). Macromol. Rapid Commun. 29, 1944-1949.

Westrip, S. P. (2010). J. Appl. Cryst. 43, 920-925.

Wickramasinhage, R., McAdam, C. J. \& Simpson, J. (2016). IUCrData, 1, x160307.

Wieczorek, M. W., Bockii, N. G. \& Struchkov, Y. T. (1975). Rocz. Chem. 49, 1737.

Wiedenfeld, D. J., Nesterov, V. N., Minton, M. A. \& Glass, D. R. (2003). Acta Cryst. C59, o700-0702. 


\section{supporting information}

Acta Cryst. (2017). E73, 658-663 [https://doi.org/10.1107/S2056989017004959]

Crystal structures of the polymer precursors 3-(2,5-dimethoxy-3,4,6-trimethylphenyl) propyl methacrylate and 3-(2,4,5-trimethyl-3,6-dioxocyclohexa-1,4dienyl)propyl methacrylate

Shailesh K. Goswami, Lyall R. Hanton, C. John McAdam, Stephen C. Moratti and Jim Simpson

Computing details

For both compounds, data collection: APEX2 (Bruker, 2013); cell refinement: APEX2 and SAINT (Bruker, 2013); data reduction: SAINT (Bruker, 2013); program(s) used to solve structure: SHELXS2013 (Sheldrick, 2008); program(s) used to refine structure: SHELXL2014/7 (Sheldrick, 2015) and TITAN (Hunter \& Simpson, 1999); molecular graphics: Mercury (Macrae et al., 2008); software used to prepare material for publication: SHELXL2014/7 (Sheldrick, 2015), enCIFer (Allen et al., 2004), PLATON (Spek, 2009), publCIF (Westrip 2010).

(I) 3-(2,5-Dimethoxy-3,4,6-trimethylphenyl) propyl methacrylate

\section{Crystal data}

$\mathrm{C}_{18} \mathrm{H}_{26} \mathrm{O}_{4}$

$M_{r}=306.39$

Monoclinic, $P 2_{1} / n$

$a=5.1833(7) \AA$

$b=30.341(4) \AA$

$c=10.6339(15) \AA$

$\beta=97.910(9)^{\circ}$

$V=1656.4(4) \AA^{3}$

$Z=4$

\section{Data collection}

Bruker APEXII CCD area detector diffractometer

Radiation source: fine-focus sealed tube Graphite monochromator $\omega$ scans

Absorption correction: multi-scan

(SADABS; Bruker, 2013)

$T_{\min }=0.775, T_{\max }=1.00$

Refinement

Refinement on $F^{2}$

Least-squares matrix: full

$R\left[F^{2}>2 \sigma\left(F^{2}\right)\right]=0.052$

$w R\left(F^{2}\right)=0.138$

$S=1.03$

1681 reflections
$F(000)=664$

$D_{\mathrm{x}}=1.229 \mathrm{Mg} \mathrm{m}^{-3}$

Mo $K \alpha$ radiation, $\lambda=0.71073 \AA$

Cell parameters from 1289 reflections

$\theta=4.7-40.3^{\circ}$

$\mu=0.09 \mathrm{~mm}^{-1}$

$T=91 \mathrm{~K}$

Needle, colourless

$0.65 \times 0.04 \times 0.04 \mathrm{~mm}$

11585 measured reflections

1681 independent reflections

1254 reflections with $I>2 \sigma(I)$

$R_{\text {int }}=0.081$

$\theta_{\max }=20.7^{\circ}, \theta_{\min }=2.4^{\circ}$

$h=-5 \rightarrow 5$

$k=-29 \rightarrow 29$

$l=-10 \rightarrow 10$

203 parameters

0 restraints

Hydrogen site location: inferred from neighbouring sites

$\mathrm{H}$-atom parameters constrained 
$w=1 /\left[\sigma^{2}\left(F_{\mathrm{o}}^{2}\right)+(0.0683 P)^{2}+1.3102 P\right]$

where $P=\left(F_{\mathrm{o}}^{2}+2 F_{\mathrm{c}}^{2}\right) / 3$

$(\Delta / \sigma)_{\max }<0.001$

$$
\Delta \rho_{\max }=0.34 \mathrm{e} \AA^{-3}
$$

\section{Special details}

Geometry. All esds (except the esd in the dihedral angle between two 1.s. planes) are estimated using the full covariance matrix. The cell esds are taken into account individually in the estimation of esds in distances, angles and torsion angles; correlations between esds in cell parameters are only used when they are defined by crystal symmetry. An approximate (isotropic) treatment of cell esds is used for estimating esds involving 1.s. planes.

Refinement. One low angle reflection with $\mathrm{Fo}<<\mathrm{Fc}$, that may have been affected by the beam-stop, was omitted from the final refinement cycles.

Fractional atomic coordinates and isotropic or equivalent isotropic displacement parameters $\left(\AA^{2}\right)$

\begin{tabular}{|c|c|c|c|c|c|}
\hline & $x$ & $y$ & $z$ & $U_{\text {iso }} * / U_{\text {eq }}$ & Occ. $(<1)$ \\
\hline $\mathrm{C} 1$ & $0.5070(7)$ & $0.13988(12)$ & $0.9190(3)$ & $0.0223(10)$ & \\
\hline $\mathrm{O} 1$ & $0.6011(5)$ & $0.17928(8)$ & $0.9780(2)$ & $0.0255(7)$ & \\
\hline C111 & $0.4922(8)$ & $0.18964(12)$ & $1.0910(4)$ & $0.0324(11)$ & \\
\hline H11A & 0.3020 & 0.1872 & 1.0741 & $0.049 *$ & \\
\hline H11B & 0.5403 & 0.2198 & 1.1176 & $0.049 *$ & \\
\hline $\mathrm{H} 11 \mathrm{C}$ & 0.5595 & 0.1690 & 1.1585 & $0.049^{*}$ & \\
\hline $\mathrm{C} 2$ & $0.2886(7)$ & $0.14223(11)$ & 0.8270 & $0.0213(10)$ & \\
\hline $\mathrm{C} 21$ & $0.1425(7)$ & $0.18507(12)$ & $0.7978(4)$ & $0.0296(10)$ & \\
\hline $\mathrm{H} 21 \mathrm{~A}$ & -0.0289 & 0.1831 & 0.8265 & $0.044 *$ & \\
\hline $\mathrm{H} 21 \mathrm{~B}$ & 0.1204 & 0.1904 & 0.7061 & $0.044^{*}$ & \\
\hline $\mathrm{H} 21 \mathrm{C}$ & 0.2414 & 0.2094 & 0.8420 & $0.044^{*}$ & \\
\hline $\mathrm{C} 3$ & $0.2007(7)$ & $0.10363(12)$ & 0.7625 & $0.0198(10)$ & \\
\hline C31 & $-0.0362(7)$ & $0.10426(12)$ & 0.6621 & $0.0263(10)$ & \\
\hline H31A & -0.0762 & 0.0742 & 0.6321 & $0.039 *$ & \\
\hline H31B & -0.0008 & 0.1227 & 0.5908 & $0.039 *$ & \\
\hline $\mathrm{H} 31 \mathrm{C}$ & -0.1850 & 0.1163 & 0.6984 & $0.039 *$ & \\
\hline $\mathrm{C} 4$ & $0.3397(7)$ & $0.06474(11)$ & 0.7918 & $0.0189(10)$ & \\
\hline $\mathrm{O} 4$ & $0.2485(5)$ & $0.02580(7)$ & $0.7301(2)$ & $0.0235(7)$ & \\
\hline $\mathrm{C} 41$ & $0.3680(8)$ & $0.01709(13)$ & $0.6187(3)$ & 0.0309 (11) & \\
\hline H41A & 0.3442 & 0.0426 & 0.5617 & $0.046^{*}$ & \\
\hline H41B & 0.2869 & -0.0089 & 0.5750 & $0.046^{*}$ & \\
\hline $\mathrm{H} 41 \mathrm{C}$ & 0.5545 & 0.0116 & 0.6432 & $0.046^{*}$ & \\
\hline $\mathrm{C} 5$ & $0.5615(7)$ & $0.06238(11)$ & 0.8830 & $0.0185(9)$ & \\
\hline C51 & $0.7126(7)$ & $0.01998(11)$ & $0.9032(3)$ & $0.0240(10)$ & \\
\hline H51A & 0.8608 & 0.0242 & 0.9700 & $0.036^{*}$ & 0.5 \\
\hline H51B & 0.5991 & -0.0032 & 0.9287 & $0.036^{*}$ & 0.5 \\
\hline $\mathrm{H} 51 \mathrm{C}$ & 0.7763 & 0.0114 & 0.8241 & $0.036^{*}$ & 0.5 \\
\hline H51D & 0.6300 & -0.0026 & 0.8452 & $0.036^{*}$ & 0.5 \\
\hline H51E & 0.8918 & 0.0248 & 0.8865 & $0.036^{*}$ & 0.5 \\
\hline $\mathrm{H} 51 \mathrm{~F}$ & 0.7145 & 0.0101 & 0.9911 & $0.036^{*}$ & 0.5 \\
\hline C6 & $0.6460(7)$ & $0.10094(12)$ & $0.9490(3)$ & $0.0211(10)$ & \\
\hline $\mathrm{C} 7$ & $0.8823(7)$ & $0.10066(11)$ & $1.0496(3)$ & $0.0229(10)$ & \\
\hline H7A & 0.9480 & 0.1312 & 1.0626 & $0.028 *$ & \\
\hline H7B & 1.0210 & 0.0829 & 1.0188 & $0.028 *$ & \\
\hline
\end{tabular}




\begin{tabular}{|c|c|c|c|c|c|}
\hline $\mathrm{C} 8$ & $0.8279(7)$ & $0.08205(12)$ & $1.1773(3)$ & $0.0233(9)$ & \\
\hline H8A & 0.6784 & 0.0980 & 1.2044 & $0.028^{*}$ & \\
\hline H8B & 0.7780 & 0.0507 & 1.1660 & $0.028 *$ & \\
\hline C9 & $1.0568(7)$ & $0.08561(12)$ & $1.2801(3)$ & $0.0268(10)$ & \\
\hline H9A & 1.0197 & 0.0700 & 1.3574 & $0.032 *$ & \\
\hline H9B & 1.2130 & 0.0722 & 1.2517 & $0.032 *$ & \\
\hline O9 & $1.1017(5)$ & $0.13212(8)$ & $1.3071(2)$ & $0.0266(7)$ & \\
\hline $\mathrm{C} 10$ & $1.3096(8)$ & $0.14204(13)$ & $1.3916(4)$ & $0.0243(10)$ & \\
\hline $\mathrm{O} 10$ & $1.4618(5)$ & $0.11468(9)$ & $1.4395(2)$ & $0.0335(8)$ & \\
\hline $\mathrm{C} 11$ & $1.3351(7)$ & $0.19019(12)$ & $1.4190(3)$ & $0.0248(10)$ & \\
\hline $\mathrm{C} 12$ & $1.1719(8)$ & $0.21884(13)$ & 1.3569 (4) & $0.0330(11)$ & \\
\hline $\mathrm{H} 12 \mathrm{~A}$ & 1.0364 & 0.2088 & 1.2941 & $0.040^{*}$ & \\
\hline H12B & 1.1902 & 0.2494 & 1.3753 & $0.040 *$ & \\
\hline $\mathrm{C} 13$ & $1.5513(8)$ & $0.20272(14)$ & $1.5203(4)$ & $0.0391(11)$ & \\
\hline $\mathrm{H} 13 \mathrm{~A}$ & 1.6460 & 0.1762 & 1.5525 & $0.059^{*}$ & 0.5 \\
\hline H13B & 1.6707 & 0.2228 & 1.4850 & $0.059^{*}$ & 0.5 \\
\hline $\mathrm{H} 13 \mathrm{C}$ & 1.4791 & 0.2174 & 1.5898 & $0.059^{*}$ & 0.5 \\
\hline H13D & 1.5512 & 0.2347 & 1.5324 & $0.059^{*}$ & 0.5 \\
\hline H13E & 1.5265 & 0.1881 & 1.5999 & $0.059^{*}$ & 0.5 \\
\hline $\mathrm{H} 13 \mathrm{~F}$ & 1.7181 & 0.1936 & 1.4951 & $0.059^{*}$ & 0.5 \\
\hline
\end{tabular}

Atomic displacement parameters $\left(\AA^{2}\right)$

\begin{tabular}{lllllll}
\hline & $U^{11}$ & $U^{22}$ & $U^{33}$ & $U^{12}$ & $U^{13}$ & $U^{23}$ \\
\hline C1 & $0.027(2)$ & $0.023(2)$ & $0.020(2)$ & $-0.006(2)$ & $0.012(2)$ & $-0.0020(18)$ \\
O1 & $0.0321(16)$ & $0.0217(16)$ & $0.0244(16)$ & $-0.0032(12)$ & $0.0100(13)$ & $-0.0026(12)$ \\
C111 & $0.042(3)$ & $0.030(3)$ & $0.028(3)$ & $0.002(2)$ & $0.013(2)$ & $-0.0046(19)$ \\
C2 & $0.022(2)$ & $0.022(2)$ & $0.022(2)$ & $0.0017(19)$ & $0.010(2)$ & $0.0053(18)$ \\
C21 & $0.025(2)$ & $0.030(2)$ & $0.034(2)$ & $0.0009(19)$ & $0.0036(19)$ & $0.0006(19)$ \\
C3 & $0.022(2)$ & $0.026(2)$ & $0.013(2)$ & $-0.0025(19)$ & $0.0075(18)$ & $-0.0001(18)$ \\
C31 & $0.031(3)$ & $0.027(2)$ & $0.022(2)$ & $-0.0015(19)$ & $0.007(2)$ & $0.0011(17)$ \\
C4 & $0.026(2)$ & $0.017(2)$ & $0.015(2)$ & $-0.0043(19)$ & $0.009(2)$ & $-0.0017(17)$ \\
O4 & $0.0286(15)$ & $0.0231(15)$ & $0.0205(16)$ & $-0.0024(13)$ & $0.0097(12)$ & $-0.0029(12)$ \\
C41 & $0.039(3)$ & $0.032(3)$ & $0.024(3)$ & $-0.003(2)$ & $0.014(2)$ & $-0.0054(19)$ \\
C5 & $0.017(2)$ & $0.020(2)$ & $0.020(2)$ & $0.0001(18)$ & $0.0082(19)$ & $0.0013(18)$ \\
C51 & $0.025(2)$ & $0.024(2)$ & $0.025(2)$ & $-0.0010(18)$ & $0.0067(18)$ & $-0.0010(17)$ \\
C6 & $0.022(2)$ & $0.024(2)$ & $0.019(2)$ & $-0.0029(19)$ & $0.0097(19)$ & $0.0001(18)$ \\
C7 & $0.026(2)$ & $0.020(2)$ & $0.023(2)$ & $0.0012(18)$ & $0.0058(19)$ & $0.0003(17)$ \\
C8 & $0.026(2)$ & $0.023(2)$ & $0.021(2)$ & $-0.0020(19)$ & $0.0056(18)$ & $0.0022(18)$ \\
C9 & $0.034(2)$ & $0.022(2)$ & $0.025(2)$ & $-0.004(2)$ & $0.0046(19)$ & $-0.0003(19)$ \\
O9 & $0.0300(17)$ & $0.0213(16)$ & $0.0274(16)$ & $-0.0022(12)$ & $0.0001(14)$ & $-0.0027(12)$ \\
C10 & $0.024(3)$ & $0.033(3)$ & $0.018(2)$ & $-0.002(2)$ & $0.011(2)$ & $-0.002(2)$ \\
O10 & $0.0331(17)$ & $0.0316(17)$ & $0.0347(18)$ & $0.0013(15)$ & $0.0005(14)$ & $0.0017(14)$ \\
C11 & $0.025(2)$ & $0.027(3)$ & $0.024(2)$ & $0.001(2)$ & $0.009(2)$ & $-0.0012(19)$ \\
C12 & $0.036(3)$ & $0.026(3)$ & $0.037(3)$ & $-0.006(2)$ & $0.008(2)$ & $-0.011(2)$ \\
C13 & $0.039(3)$ & $0.037(3)$ & $0.041(3)$ & $-0.008(2)$ & $0.005(2)$ & $-0.007(2)$ \\
& & & & & & \\
& & & & & & \\
& & & & & & \\
& & & & &
\end{tabular}


Geometric parameters $\left(\AA,{ }^{\circ}\right)$

\begin{tabular}{|c|c|c|c|}
\hline $\mathrm{C} 1-\mathrm{C} 2$ & $1.393(5)$ & $\mathrm{C} 51-\mathrm{H} 51 \mathrm{C}$ & 0.9800 \\
\hline $\mathrm{C} 1-\mathrm{C} 6$ & $1.398(5)$ & C51-H51D & 0.9800 \\
\hline $\mathrm{C} 1-\mathrm{O} 1$ & $1.406(4)$ & $\mathrm{C} 51-\mathrm{H} 51 \mathrm{E}$ & 0.9800 \\
\hline $\mathrm{O} 1-\mathrm{C} 111$ & $1.431(4)$ & $\mathrm{C} 51-\mathrm{H} 51 \mathrm{~F}$ & 0.9800 \\
\hline C111-H11A & 0.9800 & $\mathrm{C} 6-\mathrm{C} 7$ & $1.512(5)$ \\
\hline C111-H11B & 0.9800 & $\mathrm{C} 7-\mathrm{C} 8$ & $1.532(5)$ \\
\hline $\mathrm{C} 111-\mathrm{H} 11 \mathrm{C}$ & 0.9800 & $\mathrm{C} 7-\mathrm{H} 7 \mathrm{~A}$ & 0.9900 \\
\hline $\mathrm{C} 2-\mathrm{C} 3$ & $1.401(5)$ & $\mathrm{C} 7-\mathrm{H} 7 \mathrm{~B}$ & 0.9900 \\
\hline $\mathrm{C} 2-\mathrm{C} 21$ & $1.515(5)$ & $\mathrm{C} 8-\mathrm{C} 9$ & $1.503(5)$ \\
\hline $\mathrm{C} 21-\mathrm{H} 21 \mathrm{~A}$ & 0.9800 & $\mathrm{C} 8-\mathrm{H} 8 \mathrm{~A}$ & 0.9900 \\
\hline $\mathrm{C} 21-\mathrm{H} 21 \mathrm{~B}$ & 0.9800 & $\mathrm{C} 8-\mathrm{H} 8 \mathrm{~B}$ & 0.9900 \\
\hline $\mathrm{C} 21-\mathrm{H} 21 \mathrm{C}$ & 0.9800 & $\mathrm{C} 9-\mathrm{O} 9$ & $1.452(4)$ \\
\hline $\mathrm{C} 3-\mathrm{C} 4$ & $1.395(5)$ & C9-H9A & 0.9900 \\
\hline $\mathrm{C} 3-\mathrm{C} 31$ & $1.512(5)$ & C9-H9B & 0.9900 \\
\hline $\mathrm{C} 31-\mathrm{H} 31 \mathrm{~A}$ & 0.9800 & $\mathrm{O} 9-\mathrm{C} 10$ & $1.339(4)$ \\
\hline $\mathrm{C} 31-\mathrm{H} 31 \mathrm{~B}$ & 0.9800 & $\mathrm{C} 10-\mathrm{O} 10$ & $1.209(4)$ \\
\hline $\mathrm{C} 31-\mathrm{H} 31 \mathrm{C}$ & 0.9800 & $\mathrm{C} 10-\mathrm{C} 11$ & $1.492(5)$ \\
\hline $\mathrm{C} 4-\mathrm{C} 5$ & $1.400(5)$ & $\mathrm{C} 11-\mathrm{C} 12$ & $1.324(5)$ \\
\hline $\mathrm{C} 4-\mathrm{O} 4$ & $1.402(4)$ & $\mathrm{C} 11-\mathrm{C} 13$ & $1.493(5)$ \\
\hline $\mathrm{O} 4-\mathrm{C} 41$ & $1.435(4)$ & $\mathrm{C} 12-\mathrm{H} 12 \mathrm{~A}$ & 0.9500 \\
\hline $\mathrm{C} 41-\mathrm{H} 41 \mathrm{~A}$ & 0.9800 & $\mathrm{C} 12-\mathrm{H} 12 \mathrm{~B}$ & 0.9500 \\
\hline $\mathrm{C} 41-\mathrm{H} 41 \mathrm{~B}$ & 0.9800 & $\mathrm{C} 13-\mathrm{H} 13 \mathrm{~A}$ & 0.9800 \\
\hline $\mathrm{C} 41-\mathrm{H} 41 \mathrm{C}$ & 0.9800 & C13-H13B & 0.9800 \\
\hline $\mathrm{C} 5-\mathrm{C} 6$ & $1.403(5)$ & $\mathrm{C} 13-\mathrm{H} 13 \mathrm{C}$ & 0.9800 \\
\hline $\mathrm{C} 5-\mathrm{C} 51$ & $1.506(5)$ & C13-H13D & 0.9800 \\
\hline C51-H51A & 0.9800 & C13-H13E & 0.9800 \\
\hline C51-H51B & 0.9800 & $\mathrm{C} 13-\mathrm{H} 13 \mathrm{~F}$ & 0.9800 \\
\hline $\mathrm{C} 2-\mathrm{C} 1-\mathrm{C} 6$ & $123.2(3)$ & $\mathrm{H} 51 \mathrm{~A}-\mathrm{C} 51-\mathrm{H} 51 \mathrm{~F}$ & 56.3 \\
\hline $\mathrm{C} 2-\mathrm{C} 1-\mathrm{O} 1$ & $118.0(3)$ & $\mathrm{H} 51 \mathrm{~B}-\mathrm{C} 51-\mathrm{H} 51 \mathrm{~F}$ & 56.3 \\
\hline $\mathrm{C} 6-\mathrm{C} 1-\mathrm{O} 1$ & $118.7(3)$ & $\mathrm{H} 51 \mathrm{C}-\mathrm{C} 51-\mathrm{H} 51 \mathrm{~F}$ & 141.1 \\
\hline $\mathrm{C} 1-\mathrm{O} 1-\mathrm{C} 111$ & $114.2(3)$ & $\mathrm{H} 51 \mathrm{D}-\mathrm{C} 51-\mathrm{H} 51 \mathrm{~F}$ & 109.5 \\
\hline $\mathrm{O} 1-\mathrm{C} 111-\mathrm{H} 11 \mathrm{~A}$ & 109.5 & $\mathrm{H} 51 \mathrm{E}-\mathrm{C} 51-\mathrm{H} 51 \mathrm{~F}$ & 109.5 \\
\hline $\mathrm{O} 1-\mathrm{C} 111-\mathrm{H} 11 \mathrm{~B}$ & 109.5 & $\mathrm{C} 1-\mathrm{C} 6-\mathrm{C} 5$ & $118.4(3)$ \\
\hline $\mathrm{H} 11 \mathrm{~A}-\mathrm{C} 111-\mathrm{H} 11 \mathrm{~B}$ & 109.5 & $\mathrm{C} 1-\mathrm{C} 6-\mathrm{C} 7$ & $120.5(3)$ \\
\hline $\mathrm{O} 1-\mathrm{C} 111-\mathrm{H} 11 \mathrm{C}$ & 109.5 & $\mathrm{C} 5-\mathrm{C} 6-\mathrm{C} 7$ & $121.1(3)$ \\
\hline $\mathrm{H} 11 \mathrm{~A}-\mathrm{C} 111-\mathrm{H} 11 \mathrm{C}$ & 109.5 & $\mathrm{C} 6-\mathrm{C} 7-\mathrm{C} 8$ & $113.6(3)$ \\
\hline $\mathrm{H} 11 \mathrm{~B}-\mathrm{C} 111-\mathrm{H} 11 \mathrm{C}$ & 109.5 & $\mathrm{C} 6-\mathrm{C} 7-\mathrm{H} 7 \mathrm{~A}$ & 108.9 \\
\hline $\mathrm{C} 1-\mathrm{C} 2-\mathrm{C} 3$ & $118.6(3)$ & $\mathrm{C} 8-\mathrm{C} 7-\mathrm{H} 7 \mathrm{~A}$ & 108.9 \\
\hline $\mathrm{C} 1-\mathrm{C} 2-\mathrm{C} 21$ & $121.5(3)$ & $\mathrm{C} 6-\mathrm{C} 7-\mathrm{H} 7 \mathrm{~B}$ & 108.9 \\
\hline $\mathrm{C} 3-\mathrm{C} 2-\mathrm{C} 21$ & $119.8(3)$ & $\mathrm{C} 8-\mathrm{C} 7-\mathrm{H} 7 \mathrm{~B}$ & 108.9 \\
\hline $\mathrm{C} 2-\mathrm{C} 21-\mathrm{H} 21 \mathrm{~A}$ & 109.5 & $\mathrm{H} 7 \mathrm{~A}-\mathrm{C} 7-\mathrm{H} 7 \mathrm{~B}$ & 107.7 \\
\hline $\mathrm{C} 2-\mathrm{C} 21-\mathrm{H} 21 \mathrm{~B}$ & 109.5 & $\mathrm{C} 9-\mathrm{C} 8-\mathrm{C} 7$ & $113.3(3)$ \\
\hline $\mathrm{H} 21 \mathrm{~A}-\mathrm{C} 21-\mathrm{H} 21 \mathrm{~B}$ & 109.5 & $\mathrm{C} 9-\mathrm{C} 8-\mathrm{H} 8 \mathrm{~A}$ & 108.9 \\
\hline $\mathrm{C} 2-\mathrm{C} 21-\mathrm{H} 21 \mathrm{C}$ & 109.5 & $\mathrm{C} 7-\mathrm{C} 8-\mathrm{H} 8 \mathrm{~A}$ & 108.9 \\
\hline $\mathrm{H} 21 \mathrm{~A}-\mathrm{C} 21-\mathrm{H} 21 \mathrm{C}$ & 109.5 & $\mathrm{C} 9-\mathrm{C} 8-\mathrm{H} 8 \mathrm{~B}$ & 108.9 \\
\hline
\end{tabular}




\begin{tabular}{|c|c|c|c|}
\hline $\mathrm{H} 21 \mathrm{~B}-\mathrm{C} 21-\mathrm{H} 21 \mathrm{C}$ & 109.5 & $\mathrm{C} 7-\mathrm{C} 8-\mathrm{H} 8 \mathrm{~B}$ & 108.9 \\
\hline $\mathrm{C} 4-\mathrm{C} 3-\mathrm{C} 2$ & $118.3(3)$ & $\mathrm{H} 8 \mathrm{~A}-\mathrm{C} 8-\mathrm{H} 8 \mathrm{~B}$ & 107.7 \\
\hline $\mathrm{C} 4-\mathrm{C} 3-\mathrm{C} 31$ & $120.8(3)$ & $\mathrm{O} 9-\mathrm{C} 9-\mathrm{C} 8$ & $107.6(3)$ \\
\hline $\mathrm{C} 2-\mathrm{C} 3-\mathrm{C} 31$ & $120.8(3)$ & $\mathrm{O} 9-\mathrm{C} 9-\mathrm{H} 9 \mathrm{~A}$ & 110.2 \\
\hline $\mathrm{C} 3-\mathrm{C} 31-\mathrm{H} 31 \mathrm{~A}$ & 109.5 & $\mathrm{C} 8-\mathrm{C} 9-\mathrm{H} 9 \mathrm{~A}$ & 110.2 \\
\hline $\mathrm{C} 3-\mathrm{C} 31-\mathrm{H} 31 \mathrm{~B}$ & 109.5 & $\mathrm{O} 9-\mathrm{C} 9-\mathrm{H} 9 \mathrm{~B}$ & 110.2 \\
\hline $\mathrm{H} 31 \mathrm{~A}-\mathrm{C} 31-\mathrm{H} 31 \mathrm{~B}$ & 109.5 & $\mathrm{C} 8-\mathrm{C} 9-\mathrm{H} 9 \mathrm{~B}$ & 110.2 \\
\hline $\mathrm{C} 3-\mathrm{C} 31-\mathrm{H} 31 \mathrm{C}$ & 109.5 & $\mathrm{H} 9 \mathrm{~A}-\mathrm{C} 9-\mathrm{H} 9 \mathrm{~B}$ & 108.5 \\
\hline $\mathrm{H} 31 \mathrm{~A}-\mathrm{C} 31-\mathrm{H} 31 \mathrm{C}$ & 109.5 & $\mathrm{C} 10-\mathrm{O} 9-\mathrm{C} 9$ & $116.3(3)$ \\
\hline $\mathrm{H} 31 \mathrm{~B}-\mathrm{C} 31-\mathrm{H} 31 \mathrm{C}$ & 109.5 & $\mathrm{O} 10-\mathrm{C} 10-\mathrm{O} 9$ & $123.1(3)$ \\
\hline $\mathrm{C} 3-\mathrm{C} 4-\mathrm{C} 5$ & $123.2(3)$ & $\mathrm{O} 10-\mathrm{C} 10-\mathrm{C} 11$ & $123.7(4)$ \\
\hline $\mathrm{C} 3-\mathrm{C} 4-\mathrm{O} 4$ & $118.5(3)$ & $\mathrm{O} 9-\mathrm{C} 10-\mathrm{C} 11$ & $113.1(3)$ \\
\hline $\mathrm{C} 5-\mathrm{C} 4-\mathrm{O} 4$ & $118.2(3)$ & $\mathrm{C} 12-\mathrm{C} 11-\mathrm{C} 10$ & $120.8(4)$ \\
\hline $\mathrm{C} 4-\mathrm{O} 4-\mathrm{C} 41$ & $112.7(3)$ & $\mathrm{C} 12-\mathrm{C} 11-\mathrm{C} 13$ & $123.8(4)$ \\
\hline $\mathrm{O} 4-\mathrm{C} 41-\mathrm{H} 41 \mathrm{~A}$ & 109.5 & $\mathrm{C} 10-\mathrm{C} 11-\mathrm{C} 13$ & $115.3(3)$ \\
\hline $\mathrm{O} 4-\mathrm{C} 41-\mathrm{H} 41 \mathrm{~B}$ & 109.5 & $\mathrm{C} 11-\mathrm{C} 12-\mathrm{H} 12 \mathrm{~A}$ & 120.0 \\
\hline $\mathrm{H} 41 \mathrm{~A}-\mathrm{C} 41-\mathrm{H} 41 \mathrm{~B}$ & 109.5 & $\mathrm{C} 11-\mathrm{C} 12-\mathrm{H} 12 \mathrm{~B}$ & 120.0 \\
\hline $\mathrm{O} 4-\mathrm{C} 41-\mathrm{H} 41 \mathrm{C}$ & 109.5 & $\mathrm{H} 12 \mathrm{~A}-\mathrm{C} 12-\mathrm{H} 12 \mathrm{~B}$ & 120.0 \\
\hline $\mathrm{H} 41 \mathrm{~A}-\mathrm{C} 41-\mathrm{H} 41 \mathrm{C}$ & 109.5 & $\mathrm{C} 11-\mathrm{C} 13-\mathrm{H} 13 \mathrm{~A}$ & 109.5 \\
\hline $\mathrm{H} 41 \mathrm{~B}-\mathrm{C} 41-\mathrm{H} 41 \mathrm{C}$ & 109.5 & $\mathrm{C} 11-\mathrm{C} 13-\mathrm{H} 13 \mathrm{~B}$ & 109.5 \\
\hline $\mathrm{C} 4-\mathrm{C} 5-\mathrm{C} 6$ & $118.3(3)$ & $\mathrm{H} 13 \mathrm{~A}-\mathrm{C} 13-\mathrm{H} 13 \mathrm{~B}$ & 109.5 \\
\hline $\mathrm{C} 4-\mathrm{C} 5-\mathrm{C} 51$ & $120.3(3)$ & $\mathrm{C} 11-\mathrm{C} 13-\mathrm{H} 13 \mathrm{C}$ & 109.5 \\
\hline $\mathrm{C} 6-\mathrm{C} 5-\mathrm{C} 51$ & $121.3(3)$ & $\mathrm{H} 13 \mathrm{~A}-\mathrm{C} 13-\mathrm{H} 13 \mathrm{C}$ & 109.5 \\
\hline $\mathrm{C} 5-\mathrm{C} 51-\mathrm{H} 51 \mathrm{~A}$ & 109.5 & $\mathrm{H} 13 \mathrm{~B}-\mathrm{C} 13-\mathrm{H} 13 \mathrm{C}$ & 109.5 \\
\hline C5-C51-H51B & 109.5 & $\mathrm{C} 11-\mathrm{C} 13-\mathrm{H} 13 \mathrm{D}$ & 109.5 \\
\hline $\mathrm{H} 51 \mathrm{~A}-\mathrm{C} 51-\mathrm{H} 51 \mathrm{~B}$ & 109.5 & $\mathrm{H} 13 \mathrm{~A}-\mathrm{C} 13-\mathrm{H} 13 \mathrm{D}$ & 141.1 \\
\hline $\mathrm{C} 5-\mathrm{C} 51-\mathrm{H} 51 \mathrm{C}$ & 109.5 & $\mathrm{H} 13 \mathrm{~B}-\mathrm{C} 13-\mathrm{H} 13 \mathrm{D}$ & 56.3 \\
\hline $\mathrm{H} 51 \mathrm{~A}-\mathrm{C} 51-\mathrm{H} 51 \mathrm{C}$ & 109.5 & $\mathrm{H} 13 \mathrm{C}-\mathrm{C} 13-\mathrm{H} 13 \mathrm{D}$ & 56.3 \\
\hline $\mathrm{H} 51 \mathrm{~B}-\mathrm{C} 51-\mathrm{H} 51 \mathrm{C}$ & 109.5 & $\mathrm{C} 11-\mathrm{C} 13-\mathrm{H} 13 \mathrm{E}$ & 109.5 \\
\hline $\mathrm{C} 5-\mathrm{C} 51-\mathrm{H} 51 \mathrm{D}$ & 109.5 & $\mathrm{H} 13 \mathrm{~A}-\mathrm{C} 13-\mathrm{H} 13 \mathrm{E}$ & 56.3 \\
\hline $\mathrm{H} 51 \mathrm{~A}-\mathrm{C} 51-\mathrm{H} 51 \mathrm{D}$ & 141.1 & $\mathrm{H} 13 \mathrm{~B}-\mathrm{C} 13-\mathrm{H} 13 \mathrm{E}$ & 141.1 \\
\hline $\mathrm{H} 51 \mathrm{~B}-\mathrm{C} 51-\mathrm{H} 51 \mathrm{D}$ & 56.3 & $\mathrm{H} 13 \mathrm{C}-\mathrm{C} 13-\mathrm{H} 13 \mathrm{E}$ & 56.3 \\
\hline $\mathrm{H} 51 \mathrm{C}-\mathrm{C} 51-\mathrm{H} 51 \mathrm{D}$ & 56.3 & $\mathrm{H} 13 \mathrm{D}-\mathrm{C} 13-\mathrm{H} 13 \mathrm{E}$ & 109.5 \\
\hline $\mathrm{C} 5-\mathrm{C} 51-\mathrm{H} 51 \mathrm{E}$ & 109.5 & $\mathrm{C} 11-\mathrm{C} 13-\mathrm{H} 13 \mathrm{~F}$ & 109.5 \\
\hline $\mathrm{H} 51 \mathrm{~A}-\mathrm{C} 51-\mathrm{H} 51 \mathrm{E}$ & 56.3 & $\mathrm{H} 13 \mathrm{~A}-\mathrm{C} 13-\mathrm{H} 13 \mathrm{~F}$ & 56.3 \\
\hline $\mathrm{H} 51 \mathrm{~B}-\mathrm{C} 51-\mathrm{H} 51 \mathrm{E}$ & 141.1 & $\mathrm{H} 13 \mathrm{~B}-\mathrm{C} 13-\mathrm{H} 13 \mathrm{~F}$ & 56.3 \\
\hline $\mathrm{H} 51 \mathrm{C}-\mathrm{C} 51-\mathrm{H} 51 \mathrm{E}$ & 56.3 & $\mathrm{H} 13 \mathrm{C}-\mathrm{C} 13-\mathrm{H} 13 \mathrm{~F}$ & 141.1 \\
\hline $\mathrm{H} 51 \mathrm{D}-\mathrm{C} 51-\mathrm{H} 51 \mathrm{E}$ & 109.5 & $\mathrm{H} 13 \mathrm{D}-\mathrm{C} 13-\mathrm{H} 13 \mathrm{~F}$ & 109.5 \\
\hline $\mathrm{C} 5-\mathrm{C} 51-\mathrm{H} 51 \mathrm{~F}$ & 109.5 & $\mathrm{H} 13 \mathrm{E}-\mathrm{C} 13-\mathrm{H} 13 \mathrm{~F}$ & 109.5 \\
\hline $\mathrm{C} 2-\mathrm{C} 1-\mathrm{O} 1-\mathrm{C} 111$ & $90.7(4)$ & $\mathrm{C} 2-\mathrm{C} 1-\mathrm{C} 6-\mathrm{C} 5$ & $0.1(5)$ \\
\hline $\mathrm{C} 6-\mathrm{C} 1-\mathrm{O} 1-\mathrm{C} 111$ & $-93.5(4)$ & $\mathrm{O} 1-\mathrm{C} 1-\mathrm{C} 6-\mathrm{C} 5$ & $-175.5(3)$ \\
\hline $\mathrm{C} 6-\mathrm{C} 1-\mathrm{C} 2-\mathrm{C} 3$ & $1.0(5)$ & $\mathrm{C} 2-\mathrm{C} 1-\mathrm{C} 6-\mathrm{C} 7$ & $179.8(3)$ \\
\hline $\mathrm{O} 1-\mathrm{C} 1-\mathrm{C} 2-\mathrm{C} 3$ & $176.7(3)$ & $\mathrm{O} 1-\mathrm{C} 1-\mathrm{C} 6-\mathrm{C} 7$ & $4.2(5)$ \\
\hline $\mathrm{C} 6-\mathrm{C} 1-\mathrm{C} 2-\mathrm{C} 21$ & $179.6(3)$ & $\mathrm{C} 4-\mathrm{C} 5-\mathrm{C} 6-\mathrm{C} 1$ & $-1.0(5)$ \\
\hline $\mathrm{O} 1-\mathrm{C} 1-\mathrm{C} 2-\mathrm{C} 21$ & $-4.7(5)$ & $\mathrm{C} 51-\mathrm{C} 5-\mathrm{C} 6-\mathrm{C} 1$ & $175.4(3)$ \\
\hline $\mathrm{C} 1-\mathrm{C} 2-\mathrm{C} 3-\mathrm{C} 4$ & $-1.3(5)$ & $\mathrm{C} 4-\mathrm{C} 5-\mathrm{C} 6-\mathrm{C} 7$ & $179.3(3)$ \\
\hline $\mathrm{C} 21-\mathrm{C} 2-\mathrm{C} 3-\mathrm{C} 4$ & $-179.9(3)$ & $\mathrm{C} 51-\mathrm{C} 5-\mathrm{C} 6-\mathrm{C} 7$ & $-4.2(5)$ \\
\hline
\end{tabular}




$\begin{array}{llll}\mathrm{C} 1-\mathrm{C} 2-\mathrm{C} 3-\mathrm{C} 31 & 179.7(3) & \mathrm{C} 1-\mathrm{C} 6-\mathrm{C} 7-\mathrm{C} 8 & 101.6(4) \\ \mathrm{C} 21-\mathrm{C} 2-\mathrm{C} 3-\mathrm{C} 31 & 1.1(5) & \mathrm{C} 5-\mathrm{C} 6-\mathrm{C} 7-\mathrm{C} 8 & -78.7(4) \\ \mathrm{C} 2-\mathrm{C} 3-\mathrm{C} 4-\mathrm{C} 5 & 0.4(5) & \mathrm{C} 6-\mathrm{C} 7-\mathrm{C} 8-\mathrm{C} 9 & -174.8(3) \\ \mathrm{C} 31-\mathrm{C} 3-\mathrm{C} 4-\mathrm{C} 5 & 179.4(3) & \mathrm{C} 7-\mathrm{C} 8-\mathrm{C} 9-\mathrm{O} 9 & 66.7(4) \\ \mathrm{C} 2-\mathrm{C} 3-\mathrm{C} 4-\mathrm{O} 4 & 178.3(3) & \mathrm{C} 8-\mathrm{C} 9-\mathrm{O} 9-\mathrm{C} 10 & -176.0(3) \\ \mathrm{C} 31-\mathrm{C} 3-\mathrm{C} 4-\mathrm{O} 4 & -2.7(5) & \mathrm{C} 9-\mathrm{O} 9-\mathrm{C} 10-\mathrm{O} 10 & 3.5(5) \\ \mathrm{C} 3-\mathrm{C} 4-\mathrm{O} 4-\mathrm{C} 41 & 93.9(4) & \mathrm{C} 9-\mathrm{O} 9-\mathrm{C} 10-\mathrm{C} 11 & -177.2(3) \\ \mathrm{C} 5-\mathrm{C} 4-\mathrm{O} 4-\mathrm{C} 41 & -88.1(4) & \mathrm{O} 10-\mathrm{C} 10-\mathrm{C} 11-\mathrm{C} 12 & 175.3(4) \\ \mathrm{C} 3-\mathrm{C} 4-\mathrm{C} 5-\mathrm{C} 6 & 0.8(5) & \mathrm{O}-\mathrm{C} 10-\mathrm{C} 11-\mathrm{C} 12 & -4.0(5) \\ \mathrm{O} 4-\mathrm{C} 4-\mathrm{C} 5-\mathrm{C} 6 & -177.1(3) & \mathrm{O} 10-\mathrm{C} 10-\mathrm{C} 11-\mathrm{C} 13 & -5.4(5) \\ \mathrm{C} 3-\mathrm{C} 4-\mathrm{C} 5-\mathrm{C} 51 & -175.7(3) & \mathrm{O}-\mathrm{C} 10-\mathrm{C} 11-\mathrm{C} 13 & 175.3(3) \\ \mathrm{O} 4-\mathrm{C} 4-\mathrm{C} 5-\mathrm{C} 51 & 6.4(5) & & \end{array}$

Hydrogen-bond geometry $\left(\AA,{ }^{\circ}\right)$

$\mathrm{Cg}$ is the centroid of the $\mathrm{C} 1-\mathrm{C} 6$ benzene ring

\begin{tabular}{lllll}
\hline$D-\mathrm{H} \cdots A$ & $D-\mathrm{H}$ & $\mathrm{H} \cdots A$ & $D \cdots A$ & $D-\mathrm{H} \cdots A$ \\
\hline $\mathrm{C} 8-\mathrm{H} 8 B \cdots \mathrm{O} 4^{\mathrm{i}}$ & 0.99 & 2.58 & $3.456(4)$ & 147 \\
$\mathrm{C} 12-\mathrm{H} 12 B \cdots \mathrm{O} 1^{\mathrm{ii}}$ & 0.95 & 2.50 & $3.388(4)$ & 157 \\
$\mathrm{C} 21-\mathrm{H} 21 A \cdots \mathrm{O} 1^{\mathrm{iii}}$ & 0.98 & 2.67 & $3.614(5)$ & 161 \\
$\mathrm{C} 41-\mathrm{H} 41 A \cdots \mathrm{O} 10^{\mathrm{iv}}$ & 0.98 & 2.66 & $3.590(5)$ & 159 \\
$\mathrm{C} 51-\mathrm{H} 51 E \cdots 4^{\mathrm{v}}$ & 0.98 & 2.65 & $3.541(4)$ & 151 \\
$\mathrm{C} 7-\mathrm{H} 7 B \cdots C g^{\mathrm{v}}$ & 0.99 & 2.97 & $3.709(4)$ & 134 \\
$\mathrm{C} 31-\mathrm{H} 31 C \cdots C g^{\mathrm{iii}}$ & 0.98 & 2.85 & $3.693(4)$ & 148 \\
\hline
\end{tabular}

Symmetry codes: (i) $-x+1,-y,-z+2$; (ii) $x+1 / 2,-y+1 / 2, z+1 / 2$; (iii) $x-1, y, z$; (iv) $x-1, y, z-1$; (v) $x+1, y, z$.

(II) 3-(2,4,5-Trimethyl-3,6-dioxocyclohexa-1,4-dienyl)propyl methacrylate

Crystal data

$\mathrm{C}_{16} \mathrm{H}_{20} \mathrm{O}_{4}$

$M_{r}=276.32$

Monoclinic, $P 2_{1} / n$

$a=4.4096(2) \AA$

$b=11.8425(6) \AA$

$c=28.2511(16) \AA$

$\beta=93.495(3)^{\circ}$

$V=1472.55(13) \AA^{3}$

$Z=4$

\section{Data collection}

Bruker APEXII CCD area detector diffractometer

Radiation source: fine-focus sealed tube $\omega$ scans

Absorption correction: multi-scan

(SADABS; Bruker, 2013)

$T_{\text {min }}=0.785, T_{\max }=1.000$

16398 measured reflections
$F(000)=592$

$D_{\mathrm{x}}=1.246 \mathrm{Mg} \mathrm{m}^{-3}$

Mo $K \alpha$ radiation, $\lambda=0.71073 \AA$

Cell parameters from 1562 reflections

$\theta=2.3-21.1^{\circ}$

$\mu=0.09 \mathrm{~mm}^{-1}$

$T=89 \mathrm{~K}$

Irregular fragment, colourless

$0.27 \times 0.14 \times 0.13 \mathrm{~mm}$

2509 independent reflections

1774 reflections with $I>2 \sigma(I)$

$R_{\text {int }}=0.071$

$\theta_{\max }=24.8^{\circ}, \theta_{\min }=2.9^{\circ}$

$h=-5 \rightarrow 5$

$k=-13 \rightarrow 13$

$l=-31 \rightarrow 33$ 


\section{Refinement}

Refinement on $F^{2}$

Least-squares matrix: full

$R\left[F^{2}>2 \sigma\left(F^{2}\right)\right]=0.049$

$w R\left(F^{2}\right)=0.138$

$S=1.04$

2509 reflections

185 parameters

0 restraints
Hydrogen site location: inferred from neighbouring sites

$\mathrm{H}$-atom parameters constrained

$w=1 /\left[\sigma^{2}\left(F_{\mathrm{o}}^{2}\right)+(0.0609 P)^{2}+0.8066 P\right]$

where $P=\left(F_{\mathrm{o}}^{2}+2 F_{\mathrm{c}}^{2}\right) / 3$

$(\Delta / \sigma)_{\max }<0.001$

$\Delta \rho_{\max }=0.34$ e $\AA^{-3}$

$\Delta \rho_{\min }=-0.32$ e $\AA^{-3}$

Special details

Geometry. All esds (except the esd in the dihedral angle between two 1.s. planes) are estimated using the full covariance matrix. The cell esds are taken into account individually in the estimation of esds in distances, angles and torsion angles; correlations between esds in cell parameters are only used when they are defined by crystal symmetry. An approximate (isotropic) treatment of cell esds is used for estimating esds involving l.s. planes.

Fractional atomic coordinates and isotropic or equivalent isotropic displacement parameters $\left(\AA^{2}\right)$

\begin{tabular}{|c|c|c|c|c|}
\hline & $x$ & $y$ & $z$ & $U_{\text {iso }} * / U_{\text {eq }}$ \\
\hline $\mathrm{C} 1$ & $0.1775(5)$ & $0.16524(19)$ & $0.14048(8)$ & $0.0209(5)$ \\
\hline O1 & $0.0812(4)$ & $0.09197(14)$ & $0.11320(6)$ & $0.0324(5)$ \\
\hline $\mathrm{C} 2$ & $0.3994(5)$ & $0.13528(19)$ & $0.18042(8)$ & $0.0200(5)$ \\
\hline $\mathrm{C} 21$ & $0.4744(6)$ & 0.01196 (19) & $0.18425(8)$ & $0.0277(6)$ \\
\hline $\mathrm{H} 21 \mathrm{~A}$ & 0.6438 & 0.0010 & 0.2079 & $0.042 *$ \\
\hline H21B & 0.5321 & -0.0160 & 0.1534 & $0.042 *$ \\
\hline $\mathrm{H} 21 \mathrm{C}$ & 0.2964 & -0.0297 & 0.1939 & $0.042 *$ \\
\hline $\mathrm{C} 3$ & $0.5109(5)$ & $0.21672(19)$ & $0.20975(8)$ & $0.0200(5)$ \\
\hline C31 & $0.7283(6)$ & $0.1988(2)$ & $0.25224(8)$ & $0.0273(6)$ \\
\hline $\mathrm{H} 31 \mathrm{~A}$ & 0.6208 & 0.2090 & 0.2813 & $0.041 *$ \\
\hline H31B & 0.8945 & 0.2537 & 0.2516 & $0.041 *$ \\
\hline $\mathrm{H} 31 \mathrm{C}$ & 0.8113 & 0.1221 & 0.2515 & $0.041 *$ \\
\hline $\mathrm{C} 4$ & $0.4110(5)$ & $0.33563(19)$ & $0.20215(8)$ & $0.0211(5)$ \\
\hline $\mathrm{O} 4$ & $0.5126(4)$ & $0.40980(14)$ & $0.22879(6)$ & $0.0306(4)$ \\
\hline $\mathrm{C} 5$ & $0.1881(5)$ & $0.36569(18)$ & $0.16246(8)$ & $0.0194(5)$ \\
\hline C51 & $0.1114(6)$ & $0.48890(19)$ & $0.15805(9)$ & $0.0280(6)$ \\
\hline H51A & -0.0626 & 0.4987 & 0.1351 & $0.042 *$ \\
\hline H51B & 0.2866 & 0.5302 & 0.1471 & $0.042 *$ \\
\hline $\mathrm{H} 51 \mathrm{C}$ & 0.0601 & 0.5182 & 0.1890 & $0.042 *$ \\
\hline C6 & $0.0734(5)$ & $0.28445(18)$ & $0.13349(8)$ & $0.0189(5)$ \\
\hline $\mathrm{C} 7$ & $-0.1420(5)$ & $0.3062(2)$ & $0.09083(8)$ & $0.0225(5)$ \\
\hline H7A & -0.2806 & 0.3690 & 0.0979 & $0.027 *$ \\
\hline H7B & -0.2666 & 0.2380 & 0.0839 & $0.027^{*}$ \\
\hline $\mathrm{C} 8$ & $0.0333(5)$ & $0.33651(19)$ & $0.04742(8)$ & $0.0210(5)$ \\
\hline H8A & 0.1784 & 0.3979 & 0.0562 & $0.025 *$ \\
\hline H8B & 0.1526 & 0.2699 & 0.0384 & $0.025^{*}$ \\
\hline C9 & $-0.1672(5)$ & $0.37361(19)$ & $0.00508(8)$ & 0.0208 \\
\hline H9A & -0.3057 & 0.4343 & 0.0145 & $0.025^{*}$ \\
\hline H9B & -0.0408 & 0.4035 & -0.0199 & $0.025 *$ \\
\hline O9 & $-0.3418(3)$ & $0.27759(12)$ & $-0.01293(5)$ & 0.0215 \\
\hline
\end{tabular}




$\begin{array}{lllll}\text { C10 } & -0.5198(5) & 0.29825(19) & -0.05223(8) & 0.0211(5) \\ \text { O10 } & -0.5414(4) & 0.39082(13) & -0.07045(5) & 0.0264(4) \\ \text { C11 } & -0.6877(5) & 0.19585(19) & -0.06980(8) & 0.0241(6) \\ \text { C12 } & -0.6423(7) & 0.0922(2) & -0.04544(10) & 0.0441(8) \\ \text { H12A } & -0.7486 & 0.0265 & -0.0564 & 0.053^{*} \\ \text { H12B } & -0.5056 & 0.0883 & -0.0182 & 0.053^{*} \\ \text { C13 } & -0.8840(6) & 0.2102(2) & -0.11027(9) & 0.0314(6) \\ \text { H13A } & -1.0002 & 0.1407 & -0.1164 & 0.047^{*} \\ \text { H13B } & -0.7650 & 0.2273 & -0.1376 & 0.047^{*} \\ \text { H13C } & -1.0239 & 0.2727 & -0.1051 & 0.047^{*}\end{array}$

Atomic displacement parameters $\left(\AA^{2}\right)$

\begin{tabular}{lllllll}
\hline & $U^{11}$ & $U^{22}$ & $U^{33}$ & $U^{12}$ & $U^{13}$ & $U^{23}$ \\
\hline C1 & $0.0196(12)$ & $0.0212(12)$ & $0.0220(13)$ & $-0.0030(9)$ & $0.0022(10)$ & $0.0003(10)$ \\
O1 & $0.0395(11)$ & $0.0232(9)$ & $0.0328(10)$ & $-0.0028(8)$ & $-0.0109(8)$ & $-0.0067(8)$ \\
C2 & $0.0204(12)$ & $0.0213(12)$ & $0.0183(12)$ & $-0.0005(9)$ & $0.0006(10)$ & $0.0029(10)$ \\
C21 & $0.0338(15)$ & $0.0207(13)$ & $0.0281(14)$ & $0.0027(10)$ & $-0.0028(11)$ & $0.0001(11)$ \\
C3 & $0.0186(12)$ & $0.0247(12)$ & $0.0167(12)$ & $-0.0011(10)$ & $0.0032(9)$ & $0.0030(10)$ \\
C31 & $0.0297(13)$ & $0.0288(14)$ & $0.0230(13)$ & $-0.0012(11)$ & $-0.0026(11)$ & $0.0019(11)$ \\
C4 & $0.0217(12)$ & $0.0229(12)$ & $0.0191(12)$ & $-0.0039(10)$ & $0.0031(10)$ & $-0.0021(10)$ \\
O4 & $0.0364(10)$ & $0.0256(9)$ & $0.0286(10)$ & $-0.0042(8)$ & $-0.0065(8)$ & $-0.0071(8)$ \\
C5 & $0.0194(12)$ & $0.0181(12)$ & $0.0208(12)$ & $0.0003(9)$ & $0.0028(10)$ & $0.0015(10)$ \\
C51 & $0.0327(15)$ & $0.0214(13)$ & $0.0296(15)$ & $0.0021(10)$ & $0.0000(11)$ & $0.0013(11)$ \\
C6 & $0.0162(11)$ & $0.0224(12)$ & $0.0183(12)$ & $0.0000(9)$ & $0.0016(9)$ & $0.0028(10)$ \\
C7 & $0.0192(12)$ & $0.0252(13)$ & $0.0229(13)$ & $-0.0008(10)$ & $0.0000(10)$ & $0.0018(10)$ \\
C8 & $0.0177(12)$ & $0.0229(12)$ & $0.0221(13)$ & $0.0005(9)$ & $-0.0016(10)$ & $-0.0006(10)$ \\
C9 & $0.0205(12)$ & $0.0195(12)$ & $0.0221(13)$ & $-0.0023(9)$ & $-0.0027(10)$ & $0.0014(10)$ \\
O9 & $0.0236(9)$ & $0.0190(8)$ & $0.0213(9)$ & $-0.0013(6)$ & $-0.0038(7)$ & $0.0000(7)$ \\
C10 & $0.0212(12)$ & $0.0225(13)$ & $0.0194(13)$ & $0.0025(10)$ & $0.0009(10)$ & $-0.0023(10)$ \\
O10 & $0.0314(10)$ & $0.0227(9)$ & $0.0244(9)$ & $-0.0005(7)$ & $-0.0049(7)$ & $0.0044(7)$ \\
C11 & $0.0277(13)$ & $0.0227(13)$ & $0.0217(13)$ & $-0.0002(10)$ & $0.0006(10)$ & $-0.0018(10)$ \\
C12 & $0.065(2)$ & $0.0248(14)$ & $0.0399(17)$ & $-0.0126(13)$ & $-0.0195(14)$ & $-0.0009(13)$ \\
C13 & $0.0295(14)$ & $0.0303(14)$ & $0.0338(15)$ & $0.0014(11)$ & $-0.0036(11)$ & $-0.0079(12)$ \\
& & & & & & \\
\hline
\end{tabular}

Geometric parameters $\left(\AA,{ }^{o}\right)$

\begin{tabular}{llll}
\hline $\mathrm{C} 1-\mathrm{O} 1$ & $1.220(3)$ & $\mathrm{C} 6-\mathrm{C} 7$ & $1.510(3)$ \\
$\mathrm{C} 1-\mathrm{C} 2$ & $1.491(3)$ & $\mathrm{C} 7-\mathrm{C} 8$ & $1.531(3)$ \\
$\mathrm{C} 1-\mathrm{C} 6$ & $1.494(3)$ & $\mathrm{C} 7-\mathrm{H} 7 \mathrm{~A}$ & 0.9900 \\
$\mathrm{C} 2-\mathrm{C} 3$ & $1.345(3)$ & $\mathrm{C} 7-\mathrm{H} 7 \mathrm{~B}$ & 0.9900 \\
$\mathrm{C} 2-\mathrm{C} 21$ & $1.500(3)$ & $\mathrm{C} 8-\mathrm{C} 9$ & $1.509(3)$ \\
$\mathrm{C} 21-\mathrm{H} 21 \mathrm{~A}$ & 0.9800 & $\mathrm{C} 8-\mathrm{H} 8 \mathrm{~A}$ & 0.9900 \\
$\mathrm{C} 21-\mathrm{H} 21 \mathrm{~B}$ & 0.9800 & $\mathrm{C} 8-\mathrm{H} 8 \mathrm{~B}$ & 0.9900 \\
$\mathrm{C} 21-\mathrm{H} 21 \mathrm{C}$ & 0.9800 & $\mathrm{C} 9-\mathrm{O} 9$ & $1.448(3)$ \\
$\mathrm{C} 3-\mathrm{C} 4$ & $1.487(3)$ & $\mathrm{C} 9-\mathrm{H} 9 \mathrm{~A}$ & 0.9900 \\
$\mathrm{C} 3-\mathrm{C} 31$ & $1.505(3)$ & $\mathrm{C} 9-\mathrm{H} 9 \mathrm{~B}$ & 0.9900 \\
$\mathrm{C} 31-\mathrm{H} 31 \mathrm{~A}$ & 0.9800 & $\mathrm{O} 9-\mathrm{C} 10$ & $1.342(3)$
\end{tabular}




\begin{tabular}{|c|c|c|c|}
\hline $\mathrm{C} 31-\mathrm{H} 31 \mathrm{~B}$ & 0.9800 & $\mathrm{C} 10-\mathrm{O} 10$ & $1.213(3)$ \\
\hline $\mathrm{C} 31-\mathrm{H} 31 \mathrm{C}$ & 0.9800 & $\mathrm{C} 10-\mathrm{C} 11$ & $1.490(3)$ \\
\hline $\mathrm{C} 4-\mathrm{O} 4$ & $1.224(3)$ & $\mathrm{C} 11-\mathrm{C} 13$ & $1.401(3)$ \\
\hline $\mathrm{C} 4-\mathrm{C} 5$ & $1.489(3)$ & $\mathrm{C} 11-\mathrm{C} 12$ & $1.416(3)$ \\
\hline $\mathrm{C} 5-\mathrm{C} 6$ & $1.342(3)$ & $\mathrm{C} 12-\mathrm{H} 12 \mathrm{~A}$ & 0.9500 \\
\hline $\mathrm{C} 5-\mathrm{C} 51$ & $1.501(3)$ & $\mathrm{C} 12-\mathrm{H} 12 \mathrm{~B}$ & 0.9500 \\
\hline $\mathrm{C} 51-\mathrm{H} 51 \mathrm{~A}$ & 0.9800 & $\mathrm{C} 13-\mathrm{H} 13 \mathrm{~A}$ & 0.9800 \\
\hline C $51-\mathrm{H} 51 \mathrm{~B}$ & 0.9800 & C13-H13B & 0.9800 \\
\hline $\mathrm{C} 51-\mathrm{H} 51 \mathrm{C}$ & 0.9800 & $\mathrm{C} 13-\mathrm{H} 13 \mathrm{C}$ & 0.9800 \\
\hline $\mathrm{O} 1-\mathrm{C} 1-\mathrm{C} 2$ & $119.7(2)$ & $\mathrm{C} 1-\mathrm{C} 6-\mathrm{C} 7$ & $116.11(19)$ \\
\hline $\mathrm{O} 1-\mathrm{C} 1-\mathrm{C} 6$ & $119.8(2)$ & $\mathrm{C} 6-\mathrm{C} 7-\mathrm{C} 8$ & $110.83(18)$ \\
\hline $\mathrm{C} 2-\mathrm{C} 1-\mathrm{C} 6$ & $120.5(2)$ & $\mathrm{C} 6-\mathrm{C} 7-\mathrm{H} 7 \mathrm{~A}$ & 109.5 \\
\hline $\mathrm{C} 3-\mathrm{C} 2-\mathrm{C} 1$ & $119.6(2)$ & $\mathrm{C} 8-\mathrm{C} 7-\mathrm{H} 7 \mathrm{~A}$ & 109.5 \\
\hline $\mathrm{C} 3-\mathrm{C} 2-\mathrm{C} 21$ & $125.7(2)$ & $\mathrm{C} 6-\mathrm{C} 7-\mathrm{H} 7 \mathrm{~B}$ & 109.5 \\
\hline $\mathrm{C} 1-\mathrm{C} 2-\mathrm{C} 21$ & $114.71(19)$ & $\mathrm{C} 8-\mathrm{C} 7-\mathrm{H} 7 \mathrm{~B}$ & 109.5 \\
\hline $\mathrm{C} 2-\mathrm{C} 21-\mathrm{H} 21 \mathrm{~A}$ & 109.5 & $\mathrm{H} 7 \mathrm{~A}-\mathrm{C} 7-\mathrm{H} 7 \mathrm{~B}$ & 108.1 \\
\hline $\mathrm{C} 2-\mathrm{C} 21-\mathrm{H} 21 \mathrm{~B}$ & 109.5 & $\mathrm{C} 9-\mathrm{C} 8-\mathrm{C} 7$ & $113.78(18)$ \\
\hline $\mathrm{H} 21 \mathrm{~A}-\mathrm{C} 21-\mathrm{H} 21 \mathrm{~B}$ & 109.5 & $\mathrm{C} 9-\mathrm{C} 8-\mathrm{H} 8 \mathrm{~A}$ & 108.8 \\
\hline $\mathrm{C} 2-\mathrm{C} 21-\mathrm{H} 21 \mathrm{C}$ & 109.5 & $\mathrm{C} 7-\mathrm{C} 8-\mathrm{H} 8 \mathrm{~A}$ & 108.8 \\
\hline $\mathrm{H} 21 \mathrm{~A}-\mathrm{C} 21-\mathrm{H} 21 \mathrm{C}$ & 109.5 & $\mathrm{C} 9-\mathrm{C} 8-\mathrm{H} 8 \mathrm{~B}$ & 108.8 \\
\hline $\mathrm{H} 21 \mathrm{~B}-\mathrm{C} 21-\mathrm{H} 21 \mathrm{C}$ & 109.5 & $\mathrm{C} 7-\mathrm{C} 8-\mathrm{H} 8 \mathrm{~B}$ & 108.8 \\
\hline $\mathrm{C} 2-\mathrm{C} 3-\mathrm{C} 4$ & $119.7(2)$ & $\mathrm{H} 8 \mathrm{~A}-\mathrm{C} 8-\mathrm{H} 8 \mathrm{~B}$ & 107.7 \\
\hline $\mathrm{C} 2-\mathrm{C} 3-\mathrm{C} 31$ & $125.6(2)$ & $\mathrm{O} 9-\mathrm{C} 9-\mathrm{C} 8$ & $108.86(17)$ \\
\hline $\mathrm{C} 4-\mathrm{C} 3-\mathrm{C} 31$ & $114.7(2)$ & $\mathrm{O} 9-\mathrm{C} 9-\mathrm{H} 9 \mathrm{~A}$ & 109.9 \\
\hline $\mathrm{C} 3-\mathrm{C} 31-\mathrm{H} 31 \mathrm{~A}$ & 109.5 & $\mathrm{C} 8-\mathrm{C} 9-\mathrm{H} 9 \mathrm{~A}$ & 109.9 \\
\hline $\mathrm{C} 3-\mathrm{C} 31-\mathrm{H} 31 \mathrm{~B}$ & 109.5 & $\mathrm{O} 9-\mathrm{C} 9-\mathrm{H} 9 \mathrm{~B}$ & 109.9 \\
\hline $\mathrm{H} 31 \mathrm{~A}-\mathrm{C} 31-\mathrm{H} 31 \mathrm{~B}$ & 109.5 & $\mathrm{C} 8-\mathrm{C} 9-\mathrm{H} 9 \mathrm{~B}$ & 109.9 \\
\hline $\mathrm{C} 3-\mathrm{C} 31-\mathrm{H} 31 \mathrm{C}$ & 109.5 & $\mathrm{H} 9 \mathrm{~A}-\mathrm{C} 9-\mathrm{H} 9 \mathrm{~B}$ & 108.3 \\
\hline $\mathrm{H} 31 \mathrm{~A}-\mathrm{C} 31-\mathrm{H} 31 \mathrm{C}$ & 109.5 & $\mathrm{C} 10-\mathrm{O} 9-\mathrm{C} 9$ & $114.78(17)$ \\
\hline $\mathrm{H} 31 \mathrm{~B}-\mathrm{C} 31-\mathrm{H} 31 \mathrm{C}$ & 109.5 & $\mathrm{O} 10-\mathrm{C} 10-\mathrm{O} 9$ & $122.9(2)$ \\
\hline $\mathrm{O} 4-\mathrm{C} 4-\mathrm{C} 3$ & $119.8(2)$ & $\mathrm{O} 10-\mathrm{C} 10-\mathrm{C} 11$ & $124.7(2)$ \\
\hline $\mathrm{O} 4-\mathrm{C} 4-\mathrm{C} 5$ & $119.5(2)$ & $\mathrm{O} 9-\mathrm{C} 10-\mathrm{C} 11$ & $112.39(19)$ \\
\hline $\mathrm{C} 3-\mathrm{C} 4-\mathrm{C} 5$ & $120.8(2)$ & $\mathrm{C} 13-\mathrm{C} 11-\mathrm{C} 12$ & $124.3(2)$ \\
\hline $\mathrm{C} 6-\mathrm{C} 5-\mathrm{C} 4$ & $119.7(2)$ & $\mathrm{C} 13-\mathrm{C} 11-\mathrm{C} 10$ & $116.3(2)$ \\
\hline $\mathrm{C} 6-\mathrm{C} 5-\mathrm{C} 51$ & $124.9(2)$ & $\mathrm{C} 12-\mathrm{C} 11-\mathrm{C} 10$ & $119.4(2)$ \\
\hline $\mathrm{C} 4-\mathrm{C} 5-\mathrm{C} 51$ & $115.5(2)$ & $\mathrm{C} 11-\mathrm{C} 12-\mathrm{H} 12 \mathrm{~A}$ & 120.0 \\
\hline $\mathrm{C} 5-\mathrm{C} 51-\mathrm{H} 51 \mathrm{~A}$ & 109.5 & $\mathrm{C} 11-\mathrm{C} 12-\mathrm{H} 12 \mathrm{~B}$ & 120.0 \\
\hline $\mathrm{C} 5-\mathrm{C} 51-\mathrm{H} 51 \mathrm{~B}$ & 109.5 & $\mathrm{H} 12 \mathrm{~A}-\mathrm{C} 12-\mathrm{H} 12 \mathrm{~B}$ & 120.0 \\
\hline $\mathrm{H} 51 \mathrm{~A}-\mathrm{C} 51-\mathrm{H} 51 \mathrm{~B}$ & 109.5 & $\mathrm{C} 11-\mathrm{C} 13-\mathrm{H} 13 \mathrm{~A}$ & 109.5 \\
\hline $\mathrm{C} 5-\mathrm{C} 51-\mathrm{H} 51 \mathrm{C}$ & 109.5 & $\mathrm{C} 11-\mathrm{C} 13-\mathrm{H} 13 \mathrm{~B}$ & 109.5 \\
\hline $\mathrm{H} 51 \mathrm{~A}-\mathrm{C} 51-\mathrm{H} 51 \mathrm{C}$ & 109.5 & $\mathrm{H} 13 \mathrm{~A}-\mathrm{C} 13-\mathrm{H} 13 \mathrm{~B}$ & 109.5 \\
\hline $\mathrm{H} 51 \mathrm{~B}-\mathrm{C} 51-\mathrm{H} 51 \mathrm{C}$ & 109.5 & $\mathrm{C} 11-\mathrm{C} 13-\mathrm{H} 13 \mathrm{C}$ & 109.5 \\
\hline $\mathrm{C} 5-\mathrm{C} 6-\mathrm{C} 1$ & $119.7(2)$ & $\mathrm{H} 13 \mathrm{~A}-\mathrm{C} 13-\mathrm{H} 13 \mathrm{C}$ & 109.5 \\
\hline $\mathrm{C} 5-\mathrm{C} 6-\mathrm{C} 7$ & $124.0(2)$ & $\mathrm{H} 13 \mathrm{~B}-\mathrm{C} 13-\mathrm{H} 13 \mathrm{C}$ & 109.5 \\
\hline
\end{tabular}


Hydrogen-bond geometry $\left(A,{ }^{\circ}\right)$

$\mathrm{Cg}$ is the centroid of the $\mathrm{C} 1-\mathrm{C} 6$ ring.

\begin{tabular}{lllll}
\hline$D-\mathrm{H} \cdots A$ & $D-\mathrm{H}$ & $\mathrm{H} \cdots A$ & $D \cdots A$ & $D-\mathrm{H} \cdots A$ \\
\hline $\mathrm{C} 9-\mathrm{H} 9 A \cdots \mathrm{O} 10^{\mathrm{i}}$ & 0.99 & 2.72 & $3.624(3)$ & 153 \\
$\mathrm{C} 9-\mathrm{H} 9 B \cdots \mathrm{O} 10^{\mathrm{ii}}$ & 0.99 & 2.70 & $3.595(3)$ & 150 \\
$\mathrm{C} 12-\mathrm{H} 12 A \cdots \mathrm{O} 1^{\mathrm{iii}}$ & 0.95 & 2.53 & $3.422(3)$ & 156 \\
$\mathrm{C} 21-\mathrm{H} 21 A \cdots 4^{\text {iv }}$ & 0.98 & 2.52 & $3.455(3)$ & 161 \\
$\mathrm{C} 31-\mathrm{H} 31 C \cdots \mathrm{O} 4^{\text {iv }}$ & 0.98 & 2.68 & $3.638(3)$ & 166 \\
$\mathrm{C} 51-\mathrm{H} 51 B \cdots \mathrm{O} 10^{\mathrm{v}}$ & 0.98 & 2.67 & $3.510(3)$ & 144 \\
$\mathrm{C} 31-\mathrm{H} 31 B \cdots \mathrm{Cg}^{\mathrm{ii}}$ & 0.98 & 2.95 & $3.534(3)$ & 119 \\
\hline
\end{tabular}

Symmetry codes: (i) $-x-1,-y+1,-z$; (ii) $x+1, y, z$; (iii) $-x-1,-y,-z$; (iv) $-x+3 / 2, y-1 / 2,-z+1 / 2$; (v) $-x,-y+1,-z$. 